\title{
4-Factor-criticality of vertex-transitive graphs
}

\author{
Wuyang Sun* \\ Center for Discrete Mathematics \\ Fuzhou University \\ Fuzhou, Fujian 350108, China \\ swywuyang@163.com
}

\author{
Heping Zhang \\ School of Mathematics and Statistics \\ Lanzhou University \\ Lanzhou, Gansu 730000, China \\ zhanghp@lzu.edu.cn
}

Submitted: Nov 3, 2014; Accepted: Jun 18, 2016; Published: Jul 8, 2016

Mathematics Subject Classifications: 05C70

\begin{abstract}
A graph of order $n$ is $p$-factor-critical, where $p$ is an integer of the same parity as $n$, if the removal of any set of $p$ vertices results in a graph with a perfect matching. 1-factor-critical graphs and 2-factor-critical graphs are well-known factor-critical graphs and bicritical graphs, respectively. It is known that if a connected vertextransitive graph has odd order, then it is factor-critical, otherwise it is elementary bipartite or bicritical. In this paper, we show that a connected vertex-transitive non-bipartite graph of even order at least 6 is 4 -factor-critical if and only if its degree is at least 5 . This result implies that each connected non-bipartite Cayley graph of even order and degree at least 5 is 2-extendable.
\end{abstract}

Keywords: Vertex-transitive graph; 4-Factor-criticality; Matching; Connectivity

\section{Introduction}

Only finite and simple graphs are considered in this paper. A matching of a graph is a set of its mutually nonadjacent edges. A perfect matching of a graph is a matching covering all its vertices. A graph is called factor-critical if the removal of any one of its vertices results in a graph with a perfect matching. A graph is called bicritical if the removal of any pair of its distinct vertices results in a graph with a perfect matching. The concepts of factor-critical and bicritical graphs were introduced by Gallai [9] and by Lovász [12], respectively. In matching theory, factor-critical graphs and bicritical graphs are two basic bricks in matching structures of graphs [17]. Later on, the two concepts were generalized to the concept of $p$-factor-critical graphs by Favaron [7] and $\mathrm{Yu}$ [21], independently. A graph of order $n$ is said to be $p$-factor-critical, where $p$ is an integer of the same parity as $n$, if the removal of any $p$ vertices results in a graph with a perfect matching.

\footnotetext{
${ }^{*}$ Supported by NSFC grant 11371180 .
} 
$q$-extendable graphs was proposed by Plummer [17] in 1980. A connected graph of even order $n$ is $q$-extendable, where $q$ is an integer with $0 \leqslant q<n / 2$, if it has a perfect matching and every matching of size $q$ is contained in one of its perfect matchings. Favaron [8] showed that for $q$ even, every connected non-bipartite $q$-extendable graph is $q$-factorcritical. In $1993 \mathrm{Yu}$ [21] introduced an analogous concept for graphs of odd order. A connected graph of odd order is $q \frac{1}{2}$-extendable, if the removal of any one of its vertices results in a $q$-extendable graph.

A graph $G$ is said to be vertex-transitive if for any two vertices $x$ and $y$ in $G$ there is an automorphism $\varphi$ of $G$ such that $y=\varphi(x)$. A graph with a perfect matching is elementary if the union of its all perfect matchings forms a connected subgraphs. In [13], there is a following classic result about the factor-criticality and bicriticality of vertex-transitive graphs.

Theorem 1 ([13]). Let $G$ be a connected vertex-transitive graph of order $n$. Then

(a) $G$ is factor-critical if $n$ is odd;

(b) $G$ is either elementary bipartite or bicritical if $n$ is even.

A question arises naturally: Does a vertex-transitive non-bipartite graph has larger $p$-factor-criticality?

In fact, the $q$-extendability and $q \frac{1}{2}$-extendability of Cayley graphs, an important class of vertex-transitive graphs, have been investigated in literature. It was proved in papers $[3,4,16]$ that a connected Cayley graph of even order on an abelian group, a dihedral group or a generalized dihedral group is 2-extendable except for several circulant graphs of degree at most 4. Miklavič and Šparl [16] also showed that a connected Cayley graph on an abelian group of odd order $n \geqslant 3$ either is a cycle or is $1 \frac{1}{2}$-extendable. Chan et al. [3] raised the problem of characterizing 2-extendable Cayley graphs.

In [22], we showed that a connected vertex-transitive graph of odd order $n \geqslant 3$ is 3 -factor-critical if and only if it is not a cycle. This general result is stronger than $1 \frac{1}{2}$ extendability of Cayley graphs. In this paper, we obtain the following main result which gives a simple characterization of 4-factor-critical vertex-transitive non-bipartite graphs.

Theorem 2. Let $G$ be a connected vertex-transitive non-bipartite graph of degree $k$ and of even order at least 6 . Then $G$ is 4-factor-critical if and only if $k \geqslant 5$.

By Theorem 2, we know that all connected non-bipartite Cayley graphs of even order and of degree at least 5 is 2-extendable.

The necessity of Theorem 2 is clear. Our main task is to show the sufficiency of Theorem 2 by contradiction. Suppose that $G$ is a connected non-bipartite vertex-transitive graph $G$ of even order at least 6 and of degree at least 5 but $G$ is not 4 -factor-critical. By the $s$-restricted edge-connectivity of $G$, we find that in most cases a suitable integer $s$ can be chosen such that every $\lambda_{s}$-atom of $G$ is an imprimitive block. Then we can deduce contradictions. Some preliminary results are presented in Section 2 and some properties of $\lambda_{s}$-atoms of $G$ which are used to show their imprimitivity are proved in Section 3. Finally, we complete the proof of Theorem 2 in Section 4. 


\section{Preliminaries}

In this section, we introduce some notations and results needed in this paper.

Let $G=(V(G), E(G))$ be a graph. For $X \subseteq V(G)$, let $\bar{X}=V(G) \backslash X$. For $Y \subseteq \bar{X}$, denote by $[X, Y]$ the set of edges of $G$ with one end in $X$ and the other in $Y$. In particular, we denote $[X, \bar{X}]$ by $\nabla(X)$ and denote $|\nabla(X)|$ by $d_{G}(X)$. Denote by $N_{G}(X)$ the set of vertices in $\bar{X}$ which are ends of some edges in $\nabla(X)$. If $X=\{v\}$, then $X$ is usually written to $v$. Vertices in $N_{G}(v)$ are called the neighbors of $v$. If no confusion exists, the subscript $G$ are usually omitted. Denote by $G[X]$ the subgraph induced by $X$ and denote by $G-X$ the subgraph induced by $\bar{X}$. The set of edges in $G[X]$ is denoted by $E(X)$. Denote by $c_{0}(G)$ the number of the components of $G$ which have odd order. For a subgraph $H$ of $G$, we denote $d_{G}\left(V\left(H_{i}\right)\right)$ and $\nabla\left(V\left(H_{i}\right)\right)$ by $d_{G}\left(H_{i}\right)$ and $\nabla\left(H_{i}\right)$, respectively.

For a connected graph $G$, a subset $F \subseteq E(G)$ is said to be an edge-cut of $G$ if $G-F$ is disconnected, where $G-F$ is the graph with vertex-set $V(G)$ and edge-set $E(G) \backslash F$. The edge-connectivity of $G$ is the minimum cardinality over all the edge-cuts of $G$, denoted by $\lambda(G)$. A subset $X \subseteq V(G)$ is called a vertex-cut of $G$ if $G-X$ is disconnected. The vertex-connectivity of $G$ of order $n$, denoted by $\kappa(G)$, is $n-1$ if $G$ is the complete graph $K_{n}$ and is the minimum cardinality over all the vertex-cuts of $G$ otherwise. It is well known that $\kappa(G) \leqslant \lambda(G) \leqslant \delta(G)$, where $\delta(G)$ is the minimum vertex-degree of $G$.

There are two properties of $p$-factor-critical graphs.

Theorem 3 ([7,21]). A graph $G$ is p-factor-critical if and only if $c_{0}(G-X) \leqslant|X|-p$ for all $X \subseteq V(G)$ with $|X| \geqslant p$.

Theorem 4 ([7]). If a graph $G$ is p-factor-critical with $1 \leqslant p<|V(G)|$, then $\kappa(G) \geqslant p$ and $\lambda(G) \geqslant p+1$.

Let $X$ be a subset of $V(G)$. Denoted by $\mathscr{C}_{G-X}$ the set of the components of $G-X$. $X$ is called to be matchable to $\mathscr{C}_{G-X}$ if the bipartite graph $G_{X}$, which arises from $G$ by contracting the components in $\mathscr{C}_{G-X}$ to single vertices and deleting all the edges in $E(X)$, contains a matching covering $X$. The following general result will be used.

Theorem 5 ([5]). Every graph $G$ contains a set $X$ of vertices with the following properties: (a) $X$ is matchable to $\mathscr{C}_{G-X}$;

(b) Every component of $G-X$ is factor-critical.

Given any such set $X$, the graph $G$ contains a perfect matching if and only if $|X|=\left|\mathscr{C}_{G-X}\right|$.

The girth of a graph $G$ with a cycle is the length of a shortest cycle in $G$ and the odd girth of a non-bipartite graph $G$ is the length of a shortest odd cycle in $G$. The girth and odd girth of $G$ are denoted by $g(G)$ and $g_{0}(G)$, respectively. l-cycle means a cycle of length $l$. We present two useful lemmas as follows.

Lemma 6 ([15]). Let $G$ be a graph with $g_{0}(G)>3$. Then $|E(G)| \leqslant \frac{1}{4}|V(G)|^{2}$.

Lemma 7 ([1]). Let $G$ be a k-regular graph. If $g_{0}(G)>3$, then $|V(G)| \geqslant k g_{0}(G) / 2$.

Now we list some useful properties of vertex-transitive graphs as follows. 
Theorem 8 ([14]). Let $G$ be a connected vertex-transitive $k$-regular graph. Then $\lambda(G)=$ $k$.

Theorem 9 ([19]). Let $G$ be a connected vertex-transitive $k$-regular graph. Then $\kappa(G)>$ $\frac{2}{3} k$.

Lemma 10 ([19]). Let $G$ be a connected vertex-transitive $k$-regular graph. If $\kappa(G)<k$, then $\kappa(G)=m \tau(G)$ for some integer $m \geqslant 2$, where

$\tau(G)=\min \{\min \{|V(P)|: P$ is a component of $G-X\}: X$ is a minimum vertex-cut of $G\}$.

Lemma 11 ([19]). Let $G$ be a connected vertex-transitive $k$-regular graph with $k=4$ or 6. Then $\kappa(G)=k$.

An imprimitive block of $G$ is a proper non-empty subset $X$ of $V(G)$ such that for any automorphism $\varphi$ of $G$, either $\varphi(X)=X$ or $\varphi(X) \cap X=\emptyset$.

Lemma $12([18])$. Let $G$ be a vertex-transitive graph and $X$ be an imprimitive block of $G$. Then $G[X]$ is also vertex-transitive.

Theorem 13 ([10]). Let $G$ be a connected vertex-transitive $k$-regular graph of order $n$. Let $S$ be a subset of $V(G)$ chosen such that $\frac{1}{3}(k+1) \leqslant|S| \leqslant \frac{1}{2} n, d(S)$ is as small as possible, and, subject to these conditions, $|S|$ is as small as possible. If $d(S)<\frac{2}{9}(k+1)^{2}$, then $S$ is an imprimitive block of $G$.

Corollary 14 ([10]). Let $G$ be a connected vertex-transitive $k$-regular graph of order $n$. Let $S$ be a subset of $V(G)$ chosen such that $1<|S| \leqslant \frac{1}{2} n, d_{G}(S)$ is as small as possible, and, subject to these conditions, $|S|$ is as small as possible. If $d_{G}(S)<2(k-1)$, then $d_{G}(S)=|S| \geqslant k$ and $d_{G[S]}(v)=k-1$ for all $v \in S$.

Corollary 15. Let $G$ be a connected vertex-transitive $k$-regular graph. Suppose $g(G)>3$ or $|V(G)|<2 k$. Then $d_{G}(X) \geqslant 2 k-2$ for every $X \subseteq V(G)$ with $2 \leqslant|X| \leqslant|V(G)|-2$.

Proof. If $k=2$, then it is trivial. Now suppose $k \geqslant 3$ and that there is a subset $X \subseteq V(G)$ with $2 \leqslant|X| \leqslant|V(G)|-2$ such that $d_{G}(X)<2 k-2$. Let $S$ be a subset of $V(G)$ chosen such that $1<|S| \leqslant \frac{1}{2}|V(G)|, d_{G}(S)$ is as small as possible, and, subject to these conditions, $|S|$ is as small as possible. Then $d_{G}(S) \leqslant d_{G}(X)<2 k-2$. By Corollary 14, $d_{G}(S)=|S| \geqslant k$ and $d_{G[S]}(v)=k-1$ for all $v \in S$. As $2 k-3<\frac{2}{9}(k+1)^{2}, S$ is an imprimitive block of $G$ by Theorem 13. Then $|S|$ is a divisor of $|V(G)|$, which implies $|V(G)| \geqslant 2|S| \geqslant 2 k$. Thus $g(G)>3$. Noting that $|E(S)|=\frac{1}{2}(k-1)|S| \leqslant \frac{1}{4}|S|^{2}$ by Lemma 6 , we have $d_{G}(S)=|S| \geqslant 2 k-2$, a contradiction.

A subset $X$ of $V(G)$ is called an independent set of $G$ if any two vertices in $X$ are not adjacent. The maximum cardinality of independent sets of $G$ is the independent number of $G$, denoted by $\alpha(G)$. 
Lemma 16. Let $G$ be a non-bipartite vertex-transitive $k$-regular graph. Then $\alpha(G) \leqslant$ $\frac{1}{2}|V(G)|-\frac{k}{4}$ if $g_{0}(G)>3$, and $\alpha(G) \leqslant \frac{1}{3}|V(G)|$ if $g_{0}(G)=3$.

Proof. Let $X$ be a maximum independent set of $G$ and set $g_{0}:=g_{0}(G)$. Noting that $G$ is regular and non-bipartite, we have $|X|<|\bar{X}|$. Set $t=|\bar{X}|-|X|$. Since $G$ is vertextransitive, the number of $g_{0}$-cycles of $G$ containing any given vertex in $G$ is constant. Let $q$ be this constant number and let $m$ be the number of all the $g_{0}$-cycles of $G$. Note that each $g_{0}$-cycle of $G$ contains at most $\left(g_{0}-1\right) / 2$ vertices in $X$ and at least $\left(g_{0}+1\right) / 2$ vertices in $\bar{X}$. We have $q|X| \leqslant \frac{1}{2} m\left(g_{0}-1\right)$ and $q|\bar{X}| \geqslant \frac{1}{2} m\left(g_{0}+1\right)$, which implies $q t=q(|\bar{X}|-|X|) \geqslant m$.

We know $q|V(G)|=m g_{0}$ by the vertex-transitivity of $G$. Then $q t \geqslant m=\frac{q}{g_{0}}|V(G)|$, implying $t \geqslant \frac{|V(G)|}{g_{0}}$. If $g_{0}=3$, then $\alpha(G)=\frac{1}{2}(|V(G)|-t) \leqslant \frac{1}{3}|V(G)|$. If $g_{0}>3$, then $|V(G)| \geqslant k g_{0} / 2$ by Lemma 7 , which implies $\alpha(G)=\frac{1}{2}(|V(G)|-t) \leqslant \frac{1}{2}|V(G)|-\frac{k}{4}$.

A graph $G$ is called trivial if $|V(G)|=1$.

Lemma 17. Let $G$ be a connected vertex-transitive non-bipartite graph. Let $X$ be an independent set of $G$. Suppose that $G-X$ has $|X|-t$ trivial components, where $t$ is a positive integer. Then $g_{0}(G) \geqslant \frac{2|X|}{t}+1$.

Proof. Let $Y$ be the set of vertices in the trivial components of $G-X$ and set $g_{0}:=g_{0}(G)$. Let $n_{i, j}$ be the number of $g_{0}$-cycles of $G$ which contain exactly $i$ vertices in $X$ and $j$ vertices in $Y$. Set $s=\frac{1}{2}\left(g_{0}-1\right)$. Since $X$ and $Y$ are independent sets of $G$, each $g_{0}$-cycle of $G$ contains at most $s$ vertices in $X$ and contains less vertices in $Y$ than in $X$. Let $q$ be the number of $g_{0}$-cycles of $G$ containing any given vertex in $G$. We have $\sum_{0 \leqslant j<i \leqslant s} i n_{i, j}=q|X|$ and $\sum_{0 \leqslant j<i \leqslant s} j n_{i, j}=q|Y|=q(|X|-t)$. Then $q|X|=\sum_{0 \leqslant j<i \leqslant s} i n_{i, j} \leqslant \sum_{0 \leqslant j<i \leqslant s} s(i-$ j) $n_{i, j}=s\left(\sum_{0 \leqslant j<i \leqslant s} i n_{i, j}-\sum_{0 \leqslant j<i \leqslant s} j n_{i, j}\right)=s q t=\frac{1}{2}\left(g_{0}-1\right) q t$, which implies $g_{0} \geqslant$ $\frac{2|X|}{t}+1$.

Lemma 18. Let $G$ be a vertex-transitive graph with a triangle. Then the number of trivial components of $G-X$ is not larger than $|E(X)|$ for each subset $X \subseteq V(G)$.

Proof. Let $Y$ be the set of vertices in the trivial components of $G-X$. Suppose $|Y|>$ $|E(X)|$. Let $q$ be the number of triangles of $G$ containing any given vertex in $G$. Note that there are $q|Y|$ triangles of $G$ containing vertices in $Y$. As $|Y|>|E(X)|$, it implies that $G[X]$ has an edge $e$ which is contained in more than $q$ triangles. This means that more than $q$ triangles containing both ends of $e$, a contradiction.

Lemma 19. Let $G$ be a connected triangle-free vertex-transitive 6-regular graph of even order. Suppose that there are 3 distinct vertices with the same neighbors. Then $G$ is bipartite.

Proof. Suppose, to the contrary, that $G$ is non-bipartite. Then $g_{0}:=g_{0}(G) \geqslant 5$. Let $C=u_{0} u_{1} \ldots u_{g_{0}-1} u_{0}$ be a $g_{0}$-cycle of $G$. For any pair of vertices $u$ and $v$ in $V(C)$, we know $N(u) \neq N(v)$. So for each $u_{i} \in V(C)$ there are two distinct vertices $u_{i}^{\prime}$ and $u_{i}^{\prime \prime}$ in $\overline{V(C)}$ such that $N\left(u_{i}\right)=N\left(u_{i}^{\prime}\right)=N\left(u_{i}^{\prime \prime}\right)$ by the vertex-transitivity of $G$. Set 
$U_{i}=\left\{u_{i}, u_{i}^{\prime}, u_{i}^{\prime \prime}\right\}$. Then $U_{i}$ is an independent set of $G$ and $U_{i} \cap U_{j}=\emptyset$ for $j \neq i$. Noting that $u_{i}$ and $u_{i+1}$ are adjacent, we have that every vertex in $U_{i}$ is adjacent to every vertex in $U_{i+1}$, where $i+1$ is an arithmetic on modular $g_{0}$. Since $G$ is 6 -regular and connected, $|V(G)|=\left|\bigcup_{i=0}^{g_{0}-1} U_{i}\right|=3 g_{0}$, which implies that $|V(G)|$ is odd, a contradiction.

\section{$3 \quad \lambda_{s}$-atoms of vertex-transitive graphs}

In this section, we will introduce some properties of the $\lambda_{s}$-atoms of vertex-transitive graphs. The concept of $\lambda_{s}$-atoms $[11,20]$ of graphs is used in investigating the $s$-restricted edge-connectivity of graphs. The $s$-restricted edge-connectivity of graphs was proposed by Fàbrega and Fiol [6].

For a connected graph $G$ and some positive integer $s$, an edge-cut $F$ of $G$ is said to be an $s$-restricted edge-cut of $G$ if every component of $G-F$ has at least $s$ vertices. The minimum cardinality of $s$-restricted edge-cuts of $G$ is the s-restricted edge-connectivity of $G$, denoted by $\lambda_{s}(G)$. By the definition of $\lambda_{s}(G)$, we can see that $\lambda(G)=\lambda_{1}(G) \leqslant \lambda_{2}(G) \leqslant \lambda_{3}(G) \ldots$ as long as these parameters exists.

A proper subset $X$ of $V(G)$ is called a $\lambda_{s}$-fragment of $G$ if $\nabla(X)$ is an $s$-restricted edge-cut of $G$ with minimum cardinality. We can see that for every $\lambda_{s}$-fragment $X$ of $G, G[X]$ and $G[\bar{X}]$ are connected graphs of order at least $s$. A $\lambda_{s}$-fragment of $G$ with minimum cardinality is called a $\lambda_{s}$-atom of $G$.

Lemma 20. Let $G$ be a connected triangle-free vertex-transitive graph of degree $k \geqslant 5$. For an integer $s$ with $4 \leqslant s \leqslant 8$, suppose $\lambda_{s}(G) \leqslant 3 k$. Let $S$ be a $\lambda_{s}$-atom of $G$.

(a) For $X \subseteq V(G)$ with $|X| \geqslant s$ and $|\bar{X}| \geqslant s$, we have $d_{G}(X) \geqslant \lambda_{s}(G)$. Furthermore, $d_{G}(X)>\lambda_{s}(G)$ if $G[X]$ or $G[\bar{X}]$ is disconnected.

(b) For $A \subseteq S$ with $1 \leqslant|A| \leqslant|S|-s$, we have $d_{G[S]}(A)>\frac{1}{2} d_{G}(A)$.

(c) For each $\lambda_{s}$-atom $T$ of $G$ with $S \neq T$ and $S \cap T \neq \emptyset$, we have $d_{G}(S \cap T)+d_{G}(S \cup T) \leqslant$ $2 \lambda_{s}(G), d_{G}(S \backslash T)+d_{G}(T \backslash S) \leqslant 2 \lambda_{s}(G),|S \cap T| \leqslant s-1$ and $|S \backslash T| \leqslant s-1$.

Proof. (a) If $G[X]$ and $G[\bar{X}]$ are connected, then $\nabla(X)$ is an s-restricted edge-cut of $G$ and hence $d_{G}(X) \geqslant \lambda_{s}(G)$. Thus it only needs to show $d_{G}(X)>\lambda_{s}(G)$ if $G[X]$ or $G[\bar{X}]$ is disconnected.

Suppose that $G[X]$ is disconnected. If each component of $G[X]$ has less than 4 vertices, then $d_{G}(X)=k|X|-2|E(X)| \geqslant k|X|-2(|X|-2) \geqslant(k-2) s+4>3 k \geqslant \lambda_{s}(G)$. Then we assume that $G[X]$ has a component $H_{1}$ with at least 4 vertices. If each component of $G\left[\overline{V\left(H_{1}\right)}\right]$ has less than 4 vertices, then $d_{G}(X)>d_{G}\left(H_{1}\right)=d_{G}\left(\overline{V\left(H_{1}\right)}\right)>\lambda_{s}(G)$. Then we assume further that $G\left[\overline{V\left(H_{1}\right)}\right]$ has a component $H_{2}$ with at least 4 vertices. Noting that both $G$ and $H_{1}$ are connected, we have that $G\left[\overline{V\left(H_{2}\right)}\right]$ is connected, which implies that $\nabla\left(H_{2}\right)$ is a 4-restricted edge-cut of $G$. Noting that $\lambda(G)=k$ by Theorem 8 , we have $d_{G}(X) \geqslant \lambda(G)+d_{G}\left(H_{1}\right) \geqslant k+d\left(V\left(H_{2}\right)\right) \geqslant k+\lambda_{4}(G)$.

So $d(X)>\lambda_{4}(G)$. Next we consider the case that $5 \leqslant s \leqslant 8$. Set $\tau_{s}(G)=\min \{d(A)$ : $A \subseteq V(G), 4 \leqslant|A| \leqslant s-1\}$. Then $\lambda_{4}(G) \geqslant \min \left\{\lambda_{s}(G), \tau_{s}(G)\right\}$. For each subset $A \subseteq V(G)$ with $4 \leqslant|A| \leqslant 7$, noting that $|E(A)| \leqslant \frac{1}{4}|A|^{2}$ by Lemma 6 , we have $d(A)=$ $k|A|-2|E(A)| \geqslant k|A|-\frac{1}{2}|A|^{2}>2 k$. Hence $\tau_{s}(G)>2 k$. If $\lambda_{s}(G)>2 k$, then $d(X) \geqslant$ 
$k+\lambda_{4}(G)>k+2 k \geqslant \lambda_{s}(G)$. If $\lambda_{s}(G) \leqslant 2 k$, then, noting $\min \left\{\lambda_{s}(G), \tau_{s}(G)\right\} \leqslant \lambda_{4}(G) \leqslant$ $\lambda_{s}(G)$, we have $d(X) \geqslant k+\lambda_{4}(G)=k+\lambda_{s}(G)>\lambda_{s}(G)$.

(b) To the contrary, suppose $d_{G[S]}(A) \leqslant \frac{1}{2} d_{G}(A)$. Then $d_{G}(S \backslash A)=d_{G}(S)-\left(d_{G}(A)-\right.$ $\left.2 d_{G[S]}(A)\right) \leqslant d_{G}(S)=\lambda_{s}(G)$. By (a), $G[S \backslash A]$ and $G[\bar{S} \cup A]$ are connected. Hence $\nabla(S \backslash A)$ is an $s$-restricted edge-cut of $G$. By the minimality of $\lambda_{s}$-atoms of $G$, we have $d_{G}(S \backslash A)>\lambda_{s}(G)$, a contradiction.

(c) By the well-known submodular inequality (see [2] for example), we have that $d_{G}(S \cap T)+d_{G}(S \cup T) \leqslant d_{G}(S)+d_{G}(T)=2 \lambda_{s}(G)$ and $d_{G}(S \backslash T)+d_{G}(T \backslash S)=d_{G}(S \cap \bar{T})+$ $d_{G}(S \cup \bar{T}) \leqslant d_{G}(S)+d_{G}(\bar{T})=2 \lambda_{s}(G)$. Next we show $|S \cap T| \leqslant s-1$ and $|S \backslash T| \leqslant s-1$. Clearly, they hold if $|S|=s$. So we may assume $|S|>s$.

Suppose $|S \cap T| \geqslant s$. Then $d_{G}(S \cap T)=d_{G}(S)+2 d_{G[S]}(S \backslash T)-d_{G}(S \backslash T)>d_{G}(S)=$ $\lambda_{s}(G)$ by (b). Noting $|\overline{S \cup T}| \geqslant|V(G)|-|S|-|T|+|S \cap T| \geqslant s$, we have $d_{G}(S \cup T) \geqslant \lambda_{s}(G)$ by (a). Hence $d_{G}(S \cap T)+d_{G}(S \cup T)>2 \lambda_{s}(G)$, a contradiction. Thus $|S \cap T| \leqslant s-1$.

If $|S \backslash T|=|T \backslash S| \geqslant s$, then we can similarly obtain $d_{G}(S \backslash T)>\lambda_{s}(G)$ and $d_{G}(T \backslash S)>$ $\lambda_{s}(G)$ by (b), which implies $d_{G}(S \backslash T)+d_{G}(T \backslash S)>2 \lambda_{s}(G)$, a contradiction. Thus $|S \backslash T| \leqslant$ $s-1$.

Lemma 21. Let $G$ be a connected triangle-free vertex-transitive 5-regular graph of even order. For $s=5$ or 6 , suppose $\lambda_{s}(G)=s+9$. Then $|S| \geqslant s+5$ for a $\lambda_{s}$-atom $S$ of $G$.

Proof. Suppose, to the contrary, that $|S|<s+5$. As $s+9=d_{G}(S)=5|S|-2|E(S)|$, $|S|$ and $s$ have different parities. Hence $|S| \geqslant s+1$. By Lemma $20(\mathrm{~b}), \delta(G[S]) \geqslant 3$. If $|S|=s+1$, then $2|E(S)| \geqslant \delta(G[S])|S| \geqslant 3|S|$, which implies $d_{G}(S)=5|S|-2|E(S)| \leqslant$ $2|S|=2 s+2<s+9$, a contradiction. Thus $|S|=s+3$. Let $R$ be the set of vertices $u$ in $S$ with $d_{G[S]}(u)=3$. By Lemma $20(\mathrm{~b}), E(R)=\emptyset$. Noting $3 s+9 \leqslant \sum_{u \in S} d_{G[S]}(u)=$ $2|E(S)|=5|S|-\lambda_{s}(G)=4 s+6$, we have $|R| \geqslant|S|-(4 s+6-3 s-9)=6$. Since $s=5$ or $6, d_{G[S]}(R)=3|R| \geqslant 18>5(s-3) \geqslant d_{G[S]}(S \backslash R)$, a contradiction.

Lemma 22. Let $G$ be a bicritical graph. If $G$ is not 4-factor-critical, then there is a subset $X \subseteq V(G)$ with $|X| \geqslant 4$ such that $c_{0}(G-X)=|X|-2$ and every component of $G-X$ is factor-critical.

Proof. Since $G$ is not 4-factor-critical, there is a set $X_{1}$ of four vertices of $G$ such that $G-X_{1}$ has no perfect matchings. By Theorem $5, G-X_{1}$ has a vertex set $X_{2}$ such that $X_{2}$ is matchable to $\mathscr{C}_{G-X_{1}-X_{2}}$ and every component of $G-X_{1}-X_{2}$ is factor-critical. Set $X=X_{1} \cup X_{2}$. Then $c_{0}(G-X)=\left|\mathscr{C}_{G-X}\right|>\left|X_{2}\right|=|X|-4$. Since $G$ is bicritical, we have $c_{0}(G-X) \leqslant|X|-2$ by Theorem 3. Hence $|X|-4<c_{0}(G-X) \leqslant|X|-2$. Noting that $c_{0}(G-X)$ and $|X|$ have the same parity, we have $c_{0}(G-X)=|X|-2$.

In the rest of this section, we always suppose that $G$ is a connected non-bipartite vertex-transitive graph of degree $k \geqslant 5$ and even order, but $G$ is not 4-factor-critical. Also we always use the following notation. Let $X$ be a subset of $V(G)$ with $|X| \geqslant 4$ such that $c_{0}(G-X)=|X|-2$ and every component of $G-X$ is factor-critical. By Theorem 1 and Lemma 22, such subset $X$ exists. Let $H=H_{1}, H_{2}, \ldots, H_{p}$ be the nontrivial components of $G-X$. For a positive integer $m$, let $[m]$ denote the set $\{1,2, \ldots, m\}$. 
Lemma 23. We have $p \geqslant 1$. Furthermore, if $g(G)>3$, then

(a) $p=1$ if $\lambda_{5}(G)>2 k$,

(b) $|X| \geqslant 7$ and $|V(H)| \geqslant 9$ if $\lambda_{5}(G)>4 k-8$ and $5 \leqslant k \leqslant 6$, and

(c) $|X| \geqslant 10$ and $|V(H)| \geqslant 15$ if $\lambda_{6}(G) \geqslant 14$ and $k=5$.

Proof. If $p=0$, then $|V(G)|=2|X|-2 \geqslant 2 k-2 \geqslant 8$ and $\alpha(G) \geqslant|\bar{X}|=\frac{1}{2}|V(G)|-1>$ $\max \left\{\frac{1}{3}|V(G)|, \frac{1}{2}|V(G)|-\frac{k}{4}\right\}$, which contradicts Lemma 16. Thus $p \geqslant 1$.

Next we suppose $g(G)>3$. For each $i \in[p]$, we have $\left|V\left(H_{i}\right)\right| \geqslant 5$ as $H_{i}$ is triangle-free and factor-critical.

Suppose $\lambda_{5}(G)>2 k$. By Lemma 20(a), $d\left(H_{i}\right) \geqslant \lambda_{5}(G)$ for each $i \in[p]$. We have $2 p k<p \lambda_{5}(G) \leqslant \sum_{i=1}^{p} d\left(H_{i}\right)=d(X)-k\left(c_{0}(G-X)-p\right) \leqslant k(p+2)$, which implies $p<2$. Thus $p=1$. (a) is proved.

Suppose $\lambda_{5}(G)>4 k-8$ and $5 \leqslant k \leqslant 6$. We know $p=1$ by (a). Assume $k=6$. Notice that $G$ is non-bipartite. It follows from Lemma 19 that $|X| \geqslant 7$. As $d(H) \leqslant 3 k$ and $H$ is triangle-free and factor-critical, we have $|V(H)| \geqslant 9$. Assume next $k=5$. Notice that $|V(G)|=|V(H)|+2|X|-3 \geqslant 12$. By Lemma $20(\mathrm{a}), d(A) \geqslant \lambda_{5}(G)>12$ for every subset $A \subseteq V(G)$ with $|A|=6$, which implies that $G$ has no subgraphs which are isomorphic to the complete bipartite graph $K_{3,3}$. By the vertex-transitivity of $G$, it follows that $G$ has also no subgraphs which are isomorphic to $K_{2,5}$. So $|X| \geqslant 7$. If $E(X)=\emptyset$, then $g_{0}(G) \geqslant 7$ by Lemma 17, which implies $|V(H)| \geqslant 13$. If $E(X) \neq \emptyset$, then $d(H)=13$, which implies $|V(H)| \geqslant 9$. Hence the statement (b) holds.

Now we suppose $\lambda_{6}(G) \geqslant 14$ and $k=5$. Then $\lambda_{5}(G) \geqslant \min \left\{\lambda_{6}(G), 5 k-12\right\}=13$. We know $p=1$ by (a). By the above argument, we know $|X| \geqslant 7,|V(H)| \geqslant 9$ and that $G$ has no subgraphs which are isomorphic to $K_{2,5}$ or $K_{3,3}$. By Lemma 20 (a), $d(V(H) \cup A) \geqslant$ $\lambda_{6}(G)$ and $d(V(H) \backslash A) \geqslant \lambda_{6}(G)$ for every subset $A \subseteq V(G)$ with $|A| \leqslant 2$. It implies that $E(X)=\emptyset,|\nabla(u) \cap \nabla(H)| \leqslant 3$ for each $u \in V(G)$ and each of $X$ and $V(H)$ has at most one vertex $v$ with $|\nabla(v) \cap \nabla(H)|=3$. Set $Y=\overline{V(H) \cup X}$.

Suppose $|X|=7$. Then $X$ has one vertex $u_{1}$ with 3 neighbors in $V(H)$ and other vertices in $X$ has exactly two neighbors in $V(H)$. Choose a vertex $u_{2} \in X \backslash\left\{u_{1}\right\}$ and a vertex $u_{3} \in Y \backslash N\left(u_{1}\right)$. Since $G$ is vertex-transitive, there is an automorphism $\varphi_{1}$ of $G$ such that $\varphi_{1}\left(u_{3}\right)=u_{2}$. Noting that $\left|N(v) \cap N\left(u_{3}\right)\right| \geqslant 3$ for each $v \in Y$, we have $\varphi_{1}(Y) \subseteq X$, which implies $|\nabla(v) \cap \nabla(H)|=3$ for each $v \in N\left(u_{2}\right) \cap V(H)$, a contradiction.

Suppose $8 \leqslant|X| \leqslant 9$. Then there are two vertices $u_{4}$ and $u_{5}$ in $X$ with $\mid N\left(u_{4}\right) \cap$ $V(H) \mid=2$ and $\left|N\left(u_{5}\right) \cap V(H)\right| \leqslant 1$. Since $G$ is vertex-transitive, there is an automorphism $\varphi_{2}$ of $G$ such that $\varphi_{2}\left(u_{5}\right)=u_{4}$. Then $\varphi_{2}(Y) \cap V(H) \neq \emptyset$ and $\left|\varphi_{2}(Y) \cap Y\right| \geqslant 2$. As $G$ has no subgraphs which are isomorphic to $K_{2,5}$ or $K_{3,3}$, it follows that $\left|\varphi_{2}(X) \cap X\right| \geqslant 6$. Hence $\varphi_{2}(Y) \subseteq V(H) \cup Y$ and $\varphi_{2}(X) \subseteq V(H) \cup X$. Noting that $|\nabla(u) \cap \nabla(H)| \leqslant 3$ and $N(u) \subseteq \varphi_{2}(X)$ for each $u \in \varphi_{2}(Y) \cap V(H)$, we have $\left|\varphi_{2}(X) \cap V(H)\right| \geqslant 2$. Notice that each of $X$ and $V(H)$ has at most one vertex $v$ with $|\nabla(v) \cap \nabla(H)|=3$. We know $d_{G\left[\varphi_{2}(X \cup Y)\right]}\left(\varphi_{2}(X) \cap V(H)\right) \geqslant 3$, which implies $\left|\varphi_{2}(Y) \cap V(H)\right| \geqslant 3$. It follows that $N_{H}\left(\varphi_{2}(Y) \cap V(H)\right) \geqslant 3$. Now we have $\left|\varphi_{2}(X) \cap X\right|=6$ and $\left|\varphi_{2}(X) \cap V(H)\right|=3$ as $\left|\varphi_{2}(X)\right|=|X| \leqslant 9$. It follows that $G\left[\varphi_{2}(X \cup Y) \cap V(H)\right]$ contains a subgraph isomorphic to $K_{3,3}$ if $\left|\varphi_{2}(Y) \cap V(H)\right| \geqslant 4$ and $G\left[\varphi_{2}(X \cup Y) \backslash V(H)\right]$ contains a subgraph isomorphic to $K_{3,3}$ otherwise, a contradiction. 
Thus $|X| \geqslant 10$. Then $g_{0}(G) \geqslant 9$ by Lemma 17 . Let $C$ be a $g_{0}(H)$-cycle of $H$. Then $g_{0}(H) \geqslant g_{0}(G) \geqslant 9$ and $\left|N_{H}(v) \cap V(C)\right| \leqslant 2$ for each $v \in V(H) \backslash V(C)$. Noting $15=d(V(H))=5|V(H)|-2|E(H)|$, we can easily verify $|V(H)| \geqslant 15$. (c) is proved.

Lemma 24. Suppose $k=5, \lambda_{6}(G)=\lambda_{7}(G)=12$ and $g(G)>3$. For a $\lambda_{7}$-atom $S$ of $G$, we have that $S$ is an imprimitive block of $G$.

Proof. Suppose, to the contrary, that $S$ is not an imprimitive block of $G$. Then there is an automorphism $\varphi_{1}$ of $G$ such that $\varphi_{1}(S) \neq S$ and $\varphi_{1}(S) \cap S \neq \emptyset$. Set $T=\varphi_{1}(S)$. By Lemma 20(c), we have $|S \cap T| \leqslant 6$ and $|S \backslash T| \leqslant 6$, which implies $|S| \leqslant 12$. As $12=d(S)=5|S|-2|E(S)|,|S|$ is an even integer. By Lemma $20(\mathrm{~b}), \delta(G[S]) \geqslant 3$. For each $u \in \bar{S}$, we have $d_{G}(S \cup\{u\}) \geqslant \lambda_{6}(G)$ by Lemma 20 (a), which implies $\left|N_{G}(u) \cap S\right| \leqslant 2$. Noting that $\lambda_{6}(G) \geqslant \lambda_{5}(G) \geqslant \lambda_{4}(G) \geqslant \min \left\{4 k-8,5 k-12, \lambda_{6}(G)\right\}=12$, we have $\lambda_{5}(G)=$ $\lambda_{4}(G)=12$. By Lemma $23, p=1$. By Lemma $20\left(\right.$ a) , we have $d_{G}(H) \geqslant \lambda_{5}(G)=12$. Then either $d_{G}(H)=13$ and $|E(X)|=1$, or $d_{G}(H)=15$ and $E(X)=\emptyset$.

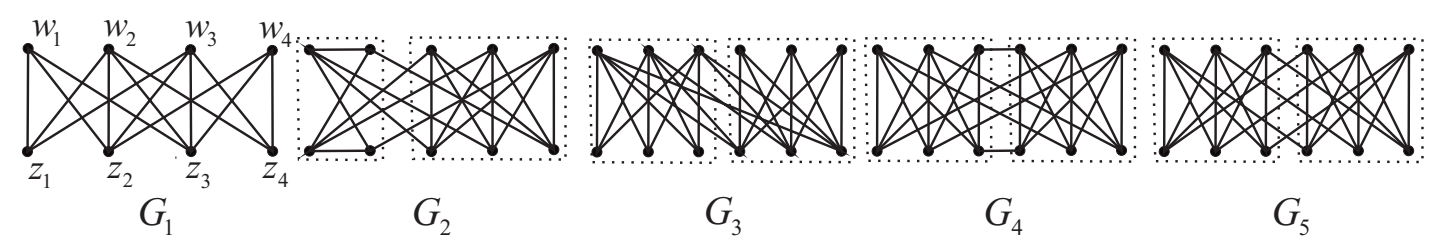

Figure 1. Some possible cases of $G[S]$. In each $G_{i}, 2 \leqslant i \leqslant 5$, the two graphs in the virtual boxes correspond to $G[S \cap T]$ and $G[S \backslash T]$.

Case 1. $|S|=8$.

We have $|E(S)|=\frac{1}{2}\left(5|S|-\lambda_{6}(G)\right)=14$. It is easy to verify that $G[S]$ is isomorphic to $G_{1}$ in Figure 1. Label $G[S]$ as in $G_{1}$ and set $W=\left\{w_{1}, w_{2}, w_{3}, w_{4}\right\}$. As $\left|N_{G}(u) \cap S\right| \leqslant 2$ for each $u \in \bar{S}, G$ has no vertex $v$ different from $w_{1}$ such that $N_{G}(v)=N_{G}\left(w_{1}\right)$. Hence $G$ has no subgraphs isomorphic to $K_{2,5}$ by the vertex-transitivity of $G$.

Claim 1. Each edge in $G$ is contained in a 4-cycle of $G$.

Suppose that $G$ has an edge contained in no 4-cycles of $G$. Since $G$ is vertex-transitive, each vertex in $G$ is incident with an edge contained in no 4-cycles of $G$ and there is an automorphism $\varphi_{2}$ of $G$ such that $\varphi_{2}\left(w_{1}\right)=w_{2}$. As each edge in $G[S]$ is contained in a 4-cycle, we have $\varphi_{2}\left(N_{G[S]}\left(w_{1}\right)\right) \subseteq N_{G[S]}\left(w_{2}\right)$ and $N_{G[S]}\left(\varphi_{2}\left(z_{i}\right)\right) \subseteq \varphi_{2}(S)$ for each $i \in\{2,3\}$. It implies $\left|S \cap \varphi_{2}(S)\right| \geqslant 7$. On the other hand, noting $\varphi_{2}(S) \neq S$, we have $\left|S \cap \varphi_{2}(S)\right| \leqslant 6$ by Lemma 20(c), a contradiction. Thus Claim 1 holds.

Claim 2. For any vertex $x \in V(G)$ with $2 \leqslant|\nabla(x) \cap \nabla(H)| \leqslant 3$ such that $d_{G[X]}(u)=0$ for each $u \in\left(\{x\} \cup N_{G}(x)\right) \cap X$, there is a subset $A \subseteq N_{G}(x)$ with $|A| \geqslant|\nabla(x) \cap \nabla(H)|-1$ and a vertex $y \in V(G) \backslash\{x\}$ such that $\{x u, y u\} \subseteq \nabla(H)$ and $|\nabla(u) \cap \nabla(H)| \geqslant 3$ for each $u \in A$.

Since $G$ is vertex-transitive, there is an automorphism $\varphi_{3}$ of $G$ such that $\varphi_{3}\left(w_{2}\right)=x$. Let $T_{1}$ be one of $X$ and $V(H)$ such that $x \in T_{1}$, and let $T_{2}$ be the other of $X$ and $V(H)$. 
Then $\varphi_{3}\left(w_{3}\right) \in T_{1}$ and $\left|\varphi_{3}\left(N_{G[S]}\left(w_{2}\right)\right) \cap T_{2}\right| \geqslant|\nabla(x) \cap \nabla(H)|-1$. If $\left|\varphi_{3}\left(N_{G[S]}\left(w_{2}\right)\right) \cap T_{2}\right| \leqslant 2$ or $\varphi_{3}(W) \subseteq T_{1}$, then we choose $A$ to be $\varphi_{3}\left(N_{G[S]}\left(w_{2}\right)\right) \cap T_{2}$. If $\left|\varphi_{3}\left(N_{G[S]}\left(w_{2}\right)\right) \cap T_{2}\right|=3$ and $\varphi_{3}(W) \backslash T_{1} \neq \emptyset$, then $\left|\varphi_{3}(W) \cap T_{1}\right|=3$ and $\left\{\varphi_{3}\left(z_{2}\right), \varphi_{3}\left(z_{3}\right)\right\} \subseteq T_{2}$. In the second case, we choose $A$ to be $\left\{\varphi_{3}\left(z_{2}\right), \varphi_{3}\left(z_{3}\right)\right\}$. Then $A$ and $\varphi_{3}\left(w_{3}\right)$ are a subset and a vertex which satisfy the condition. Thus Claim 2 holds.

Subcase 1.1. $d_{G}(H)=13$.

Let $x_{1} x_{2}$ be the edge in $E(X)$. We know $|X| \geqslant 6$ and $|V(H)| \geqslant 7$. By Lemma $20\left(\right.$ a),$d_{G}(V(H) \cup A) \geqslant \lambda_{4}(G)$ and $d_{G}(V(H) \backslash A) \geqslant \lambda_{4}(G)$ for each subset $A \subseteq V(G)$ with $|A| \leqslant 2$, which implies that $|\nabla(u) \cap \nabla(H)| \leqslant 3$ for each $u \in V(G)$ and each of $X$ and $V(H)$ has at most one vertex $v$ with $|\nabla(v) \cap \nabla(H)|=3$. Hence it follows from Claim 2 that $|\nabla(u) \cap \nabla(H)| \leqslant 2$ for each $u \in X \backslash\left\{x_{1}, x_{2}\right\}$. This together with Claim 2 implies that $|\nabla(u) \cap \nabla(H)| \leqslant 1$ for each $u \in V(H) \backslash N_{G}\left(\left\{x_{1}, x_{2}\right\}\right)$.

We claim $|\nabla(u) \cap \nabla(H)| \leqslant 2$ for each $u \in N_{G}\left(\left\{x_{1}, x_{2}\right\}\right) \cap V(H)$. Otherwise, suppose that there is a vertex $u_{0} \in N_{G}\left(\left\{x_{1}, x_{2}\right\}\right) \cap V(H)$ with $\left|\nabla\left(u_{0}\right) \cap \nabla(H)\right|=3$. Since $G$ is vertex-transitive, there is an automorphism $\varphi_{4}$ of $G$ such that $\varphi_{4}\left(w_{2}\right)=u_{0}$. It implies that there is a vertex $u_{1} \in \varphi_{4}\left(N_{G[S]}\left(w_{2}\right) \cap\left(X \backslash\left\{x_{1}, x_{2}\right\}\right)\right.$ such that $\left|\nabla\left(u_{1}\right) \cap \nabla(H)\right|=3$, a contradiction.

Thus it follows from Claim 2 that $|\nabla(u) \cap \nabla(H)| \leqslant 1$ for each $u \in X \backslash\left\{x_{1}, x_{2}\right\}$. Noting $\left|N_{G}\left(\left\{x_{1}, x_{2}\right\}\right) \cap V(H)\right| \leqslant 5$, we have $\left|\nabla\left(N_{G}\left(\left\{x_{1}, x_{2}\right\}\right) \cap V(H)\right) \cap \nabla(H)\right| \leqslant 10$ by the claim in the previous paragraph. Hence there is an edge $x_{3} x_{4} \in \nabla(H)$ such that $x_{3} \in X \backslash\left\{x_{1}, x_{2}\right\}$ and $\left|\nabla\left(x_{3}\right) \cap \nabla(H)\right|=\left|\nabla\left(x_{4}\right) \cap \nabla(H)\right|=1$. Then $x_{3} x_{4}$ is contained in no 4-cycles of $G$, contradicting Claim 1. Hence Subcase 1.1 cannot occur.

Subcase 1.2. $d_{G}(H)=15$.

Notice that $G$ has no subgraphs which are isomorphic to $K_{2,5}$. We know $|X| \geqslant 6$. Next we show $|V(H)| \geqslant 9$. Let $O_{i}$ be the set of vertices $u$ in $G$ with $|\nabla(u) \cap \nabla(H)|=i$ for $1 \leqslant i \leqslant 5$. If $|X| \geqslant 7$, then $g_{0}(G) \geqslant 7$ by Lemma 17 , which implies $|V(H)| \geqslant 13$. Then we assume $|X|=6$. As $G$ has no subgraphs which are isomorphic to $K_{2,5}$, we have $\left|O_{3} \cap X\right|=3$ and $\left|O_{2} \cap X\right|=3$. Noting $g(G)>3$, we can obtain $|V(H)| \neq 5$. By Claim 2, $\left|O_{3} \cap V(H)\right| \geqslant 2$, which implies $|V(H)| \neq 7$. Hence $|V(H)| \geqslant 9$.

By Lemma 20(a), $d_{G}(V(H) \cup A) \geqslant \lambda_{4}(G)$ and $d_{G}(V(H) \backslash A) \geqslant \lambda_{4}(G)$ for each subset $A \subseteq V(G)$ with $|A| \leqslant 4$. It implies $O_{5}=\emptyset,\left|O_{4} \cap X\right| \leqslant 1,\left|O_{3} \cap X\right| \leqslant 3,\left|O_{3} \cap V(H)\right| \leqslant 3$ and $\left|O_{4} \cap X\right| \cdot\left|O_{3} \cap X\right|=0$.

We claim $O_{4}=\emptyset$. Otherwise, suppose $O_{4} \neq \emptyset$. Noting that $\delta(H) \geqslant 2$ as $H$ is factorcritical, we have $O_{4} \subseteq X$. Now we know $\left|O_{4}\right|=1$ and $O_{3} \cap X=\emptyset$. It follows from Claim 2 that $O_{3} \cap V(H)=\emptyset$ and $O_{2} \subseteq N_{G}\left(O_{4}\right)$. As $\left|\nabla\left(N_{G}\left(O_{4}\right) \cap V(H)\right)\right| \leqslant 8$, there is an edge $x_{5} x_{6} \in \nabla(H)$ with $\left\{x_{5}, x_{6}\right\} \subseteq O_{1}$. Then $x_{5} x_{6}$ is contained in no 4-cycles of $G$, contradicting Claim 1.

Let $F_{1}$ be the subgraph of $G$ with vertex set $\bigcup_{i=1}^{3} O_{i}$ and edge set $\nabla(H)$ and let $F_{2}$ be the subgraph of $F_{1}$ which is induced by $O_{3}$. By Claim $2, \delta\left(F_{2}\right) \geqslant 2$. Hence $F_{2}$ is connected. Then $F_{1}$ is connected by Claims 1 and 2 . Let $t$ be the number of vertices $u$ in $F_{2}$ with $d_{F_{2}}(u)=2$. We have $15=\left|E\left(F_{1}\right)\right| \leqslant\left|E\left(F_{2}\right)\right|+2 t=6\left|O_{3}\right|-3\left|E\left(F_{2}\right)\right|$ by Claim 2. It follows that $\left|O_{3}\right|=6$ and $6 \leqslant\left|E\left(F_{2}\right)\right| \leqslant 7$.

Assume $\left|E\left(F_{2}\right)\right|=6$. Then $F_{2}$ is a 6 -cycle. For each $u \in O_{3} \cap X$, there is a vertex 
$y_{u} \in X \backslash O_{3}$ such that $N_{F_{2}}(u) \subseteq N_{G}\left(y_{u}\right)$ by Claim 2. It implies that there is a vertex $y \in X \backslash O_{3}$ such that $O_{3} \cap V(H) \subseteq N_{G}(y)$, which contradicts $\left|O_{3} \cap X\right| \leqslant 3$.

Assume $\left|E\left(F_{2}\right)\right|=7$. As $\left|E\left(F_{1}\right) \backslash E\left(F_{2}\right)\right|=8$, it follows from Claim 2 that there is a vertex $u_{2} \in V\left(F_{1}\right) \backslash O_{3}$ with $d_{F_{1}}\left(u_{2}\right)=2$ and we know $\left|N_{F_{1}}\left(u_{2}\right) \cap O_{3}\right|=1$ and $d_{F_{1}}\left(N_{F_{1}}\left(u_{2}\right) \backslash O_{3}\right)=1$. It is easy to see that there is no vertex $u^{\prime}$ in $G$ such that $\mid N_{G}\left(u^{\prime}\right) \cap$ $N_{G}\left(u_{2}\right) \mid=4$. Noting $\left|N_{G}\left(w_{2}\right) \cap N_{G}\left(w_{3}\right)\right|=4$, we have that there is no automorphism $\varphi$ of $G$ such that $\varphi\left(w_{2}\right)=u_{2}$, which contradicts the vertex-transitivity of $G$.

Case 2. $|S|=10$ or 12 .

Claim 3. For any given two distinct $\lambda_{7}$-atoms $S_{1}$ and $S_{2}$ of $G$ with $S_{1} \cap S_{2} \neq \emptyset$, $G\left[S_{1} \cap S_{2}\right]$ and $G\left[S_{1} \backslash S_{2}\right]$ are isomorphic to $K_{3,3}$ or $K_{2,2}$.

By Lemma 20(c), we have $d_{G}\left(S_{1} \cap S_{2}\right)+d_{G}\left(S_{1} \cup S_{2}\right) \leqslant 2 \lambda_{7}(G), d_{G}\left(S_{1} \backslash S_{2}\right)+d_{G}\left(S_{2} \backslash S_{1}\right) \leqslant$ $2 \lambda_{7}(G),\left|S_{1} \cap S_{2}\right| \leqslant 6$ and $\left|S_{1} \backslash S_{2}\right| \leqslant 6$. Then $\left|S_{1} \cap S_{2}\right| \geqslant 4$ and $\left|S_{1} \backslash S_{2}\right| \geqslant 4$. By Lemma 20(a), each of $d_{G}\left(S_{1} \cap S_{2}\right), d_{G}\left(S_{1} \cup S_{2}\right), d_{G}\left(S_{1} \backslash S_{2}\right)$ and $d_{G}\left(S_{2} \backslash S_{1}\right)$ is not less than $\lambda_{4}(G)$. Noting $\lambda_{4}(G)=\lambda_{7}(G)=12$, we have $d_{G}\left(S_{1} \cap S_{2}\right)=d_{G}\left(S_{1} \backslash S_{2}\right)=12$. Then $G\left[S_{1} \cap S_{2}\right]$ and $G\left[S_{1} \backslash S_{2}\right]$ are isomorphic to $K_{3,3}$ or $K_{2,2}$. So Claim 3 holds.

Let $R_{i}$ be the set of vertices $u$ in $S$ with $d_{G[S]}(u)=i$ for $3 \leqslant i \leqslant 5$. By Lemma 20(b), $E\left(G\left[R_{3}\right]\right)=\emptyset$.

Claim 4. $R_{5}=\emptyset$, or $G\left[R_{5}\right]$ is a 6 -cycle and $|S|=12$.

Suppose $R_{5} \neq \emptyset$. It only needs to show that $|S|=12$ and $G\left[R_{5}\right]$ is a 6-cycle. Assume $R_{4} \neq \emptyset$. Choose a vertex $u \in R_{4}$ and a vertex $v \in R_{5}$. Let $\varphi_{5}$ be an automorphism of $G$ such that $\varphi_{5}(u)=v$. Then $\varphi_{5}\left(N_{G[S]}(u)\right) \subseteq N_{G[S]}(v)$, which contradicts that $G\left[\varphi_{5}(S) \cap S\right]$ is isomorphic to $K_{3,3}$ or $K_{2,2}$ by Claim 3. Thus $R_{4}=\emptyset$. Noting $\left|R_{3}\right|+\left|R_{5}\right|=|S|$ and $3\left|R_{3}\right|+5\left|R_{5}\right|=2|E(S)|=5|S|-12$, we have $\left|R_{3}\right|=6$. For any two vertices $u^{\prime}, u^{\prime \prime} \in R_{5}$, it follows from Claim 3 that $\varphi(S)=S$ for every automorphism $\varphi$ of $G$ with $\varphi\left(u^{\prime}\right)=u^{\prime \prime}$. Hence $G\left[R_{5}\right]$ is $r$-regular, for some integer $r$. Then $18=3\left|R_{3}\right|=d_{G[S]}\left(R_{3}\right)=d_{G[S]}\left(R_{5}\right)=$ $(5-r)(|S|-6)$, which implies $|S|=12$ and $r=2$. Hence $G\left[R_{5}\right]$ is a 6-cycle and Claim 4 is proved.

By Claim 3, $G[S \cap T]$ and $G[S \backslash T]$ are isomorphic to $K_{3,3}$ or $K_{2,2}$. Noting $E\left(G\left[R_{3}\right]\right)=\emptyset$, we have by Claim 4 that $G[S]$ is isomorphic to $G_{2}, G_{3}, G_{4}$ or $G_{5}$ in Figure 1.

Claim 5. Each vertex in $G$ is contained in exactly two distinct $\lambda_{7}$-atoms of $G$.

By the vertex-transitivity of $G$, it only needs to show that $S^{\prime}=S$ or $S^{\prime}=T$ for a $\lambda_{7}$-atom $S^{\prime}$ of $G$ with $S^{\prime} \cap S \cap T \neq \emptyset$. Suppose $S^{\prime} \neq S$ and $S^{\prime} \neq T$. From Figure 1, we can see that $S$ has no subset $A$ different from $S \cap T$ and $S \backslash T$ such that $G[A]$ is isomorphic to $K_{3,3}$. Hence it follows from Claim 3 that $S^{\prime} \cap S=S \cap T=S^{\prime} \cap T$. Then $12=d_{G}(S \cap T) \geqslant d_{G[S]}(S \cap T)+d_{G[T]}(S \cap T)+d_{G\left[S^{\prime}\right]}(S \cap T)=18$, a contradiction. So Claim 5 holds.

Suppose $|S|=10$. Then $G[S]$ is isomorphic to $G_{2}$. By Claims 3 and 5 , there is a $\lambda_{7}$-atom $S^{\prime \prime}$ of $G$ such that $S^{\prime \prime} \cap S=S \backslash T$. Choose a vertex $u_{3} \in S \backslash T$ and a vertex $u_{4} \in S \cap T$. Noting that $G[S \backslash T]$ is not isomorphic to $G[S \cap T]$, we know by Claim 5 that there is no automorphism $\varphi$ of $G$ such that $\varphi\left(u_{3}\right)=u_{4}$, a contradiction. 
Suppose next $|S|=12$. Then $G[S]$ is isomorphic to $G_{3}, G_{4}$ or $G_{5}$. Let $V_{1}, V_{2}, \ldots$, $V_{m}$ be all subsets of $V(G)$ which induce subgraphs of $G$ isomorphic to $K_{3,3}$. Noting that $G[S \cap T]$ and $G[S \backslash T]$ are isomorphic to $K_{3,3}$, we can obtain by Claims 3 and 5 that $V_{1}, V_{2}, \ldots, V_{m}$ form a partition of $V(G)$ and for each $V_{i}$ there are exactly two elements $j_{1}, j_{2} \in\{1,2, \ldots, m\} \backslash\{i\}$ such that $G\left[V_{i} \cup V_{j_{1}}\right]$ and $G\left[V_{i} \cup V_{j_{2}}\right]$ are isomorphic to $G[S]$. We denote $V_{i} \sim V_{j}$ if $G\left[V_{i} \cup V_{j}\right]$ is isomorphic to $G[S]$, and assume $V_{1} \sim V_{2} \sim \cdots \sim V_{m} \sim V_{1}$. If $G[S]$ is isomorphic to $G_{3}$, then it is easy to verify that $G$ is bipartite, a contradiction. Thus $G[S]$ is isomorphic to $G_{4}$ or $G_{5}$.

Assume that there is some $V_{q} \subseteq \overline{V(H)}$. If $G[S]$ is isomorphic to $G_{4}$, then $\left|V_{q} \cap X\right|=3$ and $N_{G}\left(V_{q} \backslash X\right) \cap V_{q-1} \subseteq X$, which implies $|E(X)| \geqslant\left|E\left(V_{q-1}\right) \cap E(X)\right| \geqslant 2$, a contradiction. Thus $G[S]$ is isomorphic to $G_{5}$. Let $V_{j}$ be chosen such that $V_{j} \cap V(H) \neq \emptyset$ and $|j-q|$ is as small as possible. Then $\left|V_{j} \cap X\right|=3$ and $|N(u) \cap X| \geqslant 4$ for each $u \in V_{j} \cap V(H)$, which contradicts that $\delta(H) \geqslant 2$.

We now assume that $V_{i} \cap V(H) \neq \emptyset$ for $1 \leqslant i \leqslant m$. Then $\left|V_{i} \cap X\right|>\left|V_{i} \backslash(V(H) \cup X)\right|$ if $V_{i} \cap X \neq \emptyset$. Choose some $V_{q^{\prime}}$ which contains vertices in $V(G) \backslash(V(H) \cup X)$. Then $V_{q^{\prime}-1} \cap X \neq \emptyset$ and $V_{q^{\prime}+1} \cap X \neq \emptyset$. Noting $c_{0}(G-X)=|X|-2$, we can obtain that for each $i \in[m],\left|V_{i} \cap X\right|=\left|V_{i} \backslash(V(H) \cup X)\right|+1$ if $i \in\left\{q^{\prime}-1, q^{\prime}, q^{\prime}+1\right\}$ and $\left|V_{i} \cap X\right|=\emptyset$ otherwise. Then $\left|V_{q^{\prime}} \backslash(V(H) \cup X)\right|=2$. Hence $\left.\left.\mid V_{q^{\prime}-1} \cap X\right)|=| V_{q^{\prime}+1} \cap X\right) \mid=3$. Now we have $V_{q^{\prime}-1} \sim V_{q^{\prime}} \sim V_{q^{\prime}+1} \sim V_{q^{\prime}-1}$, which implies $V(G)=V_{q^{\prime}-1} \cup V_{q^{\prime}} \cup V_{q^{\prime}+1}$ and $|V(H)|=3$. It follows that $g(G)=3$, a contradiction.

Lemma 25. Suppose $k=5, \lambda_{5}(G)=\lambda_{6}(G)=13$ and $g(G)>3$. For a $\lambda_{6}$-atom $S$ of $G$, we have $|S| \geqslant 11$.

Proof. To the contrary, suppose $|S|<11$. As $13=d(S)=5|S|-2|E(S)|,|S|$ is odd. Then $|S| \geqslant 7$. By Lemma $20(\mathrm{~b}), \delta(G[S]) \geqslant 3$. By Lemma 23 , we have $p=1,|X| \geqslant 7$ and $|V(H)| \geqslant 9$. Hence $|V(G)| \geqslant 20$.

Assume $|S|=7$. Then $|E(S)|=\frac{1}{2}(5|S|-13)=11$. If $G[S]$ is bipartite, then $|E(S)| \geqslant \frac{1}{2}(|S|+1) \delta(G[S]) \geqslant 12$, a contradiction. Thus $G[S]$ is non-bipartite. Let $C$ be a shortest cycle of odd length in $G[S]$. Then $5 \leqslant|V(C)| \leqslant 7$. Noting that $\left|N_{G[S]}(u) \cap V(C)\right| \leqslant 2$ for each $u \in S \backslash V(C)$, we have $|E(S)| \leqslant 10$, a contradiction.

So $|S|=9$. Let $R_{i}$ be the set of vertices $u$ in $S$ with $d_{G[S]}(u)=i$ for $3 \leqslant i \leqslant 5$.

Claim 1. For any automorphism $\varphi$ of $G$ with $\varphi\left(R_{4} \cup R_{5}\right) \cap\left(R_{4} \cup R_{5}\right) \neq \emptyset$, either $\varphi(S)=S$ or $G[S \cap \varphi(S)]$ is isomorphic to $K_{2,3}$.

Suppose $\varphi(S) \neq S$. By Lemma $20(\mathrm{c}),|S \cap \varphi(S)| \leqslant 5,|S \backslash \varphi(S)| \leqslant 5$ and $d(S \cap \varphi(S))+$ $d(S \cup \varphi(S)) \leqslant 2 \lambda_{6}(G)$. Then $4 \leqslant|S \cap \varphi(S)| \leqslant 5$ and $|S \cup \varphi(S)|=|S|+|\varphi(S)|-\mid S \cap$ $\varphi(S) \mid \leqslant 14$. As $|V(G)| \geqslant 20$, we have $d(S \cup \varphi(S)) \geqslant \lambda_{6}(G)$ by Lemma 20 (a). Hence $d(S \cap \varphi(S)) \leqslant \lambda_{6}(G)=13$. This, together with the fact that $\left|N_{G[\varphi(S)]}(u) \cap N_{G[S]}(u)\right| \geqslant 3$ for each $u \in \varphi\left(R_{4} \cup R_{5}\right) \cap\left(R_{4} \cup R_{5}\right)$, implies that $G[S \cap \varphi(S)]$ is isomorphic to $K_{2,3}$. So Claim 1 holds.

By Claim 1, it follows that $G$ has no automorphism $\varphi$ such that $\varphi\left(R_{4}\right) \cap R_{5} \neq \emptyset$. It implies $R_{4}=\emptyset$ or $R_{5}=\emptyset$. Noting $\sum_{i=3}^{5} i\left|R_{i}\right|=2|E(S)|=32$ and $\sum_{i=3}^{5}\left|R_{i}\right|=|S|=9$, we have $\left|R_{3}\right|=4,\left|R_{4}\right|=5$ and $R_{5}=\emptyset$. By Lemma $20(\mathrm{~b}), E\left(R_{3}\right)=\emptyset$. Hence $\left|E\left(R_{4}\right)\right|=4$. 
As $g(G[S]) \geqslant g(G)>3$, it is easy to verify that either $G\left[R_{4}\right]$ has a 4-cycle or $G\left[R_{4}\right]$ is isomorphic to $K_{1,4}$. Let $u_{1}$ and $u_{2}$ be two vertices in $R_{4}$ with $d_{G\left[R_{4}\right]}\left(u_{1}\right)<d_{G\left[R_{4}\right]}\left(u_{2}\right)$. Since $G$ is vertex-transitive, there is an automorphism $\psi$ of $G$ such that $\psi\left(u_{2}\right)=u_{1}$. By Claim $1, G[\psi(S) \cap S]$ is isomorphic to $K_{2,3}$. As $u_{1}, u_{2} \in R_{4}$, we know $d_{G[\psi(S) \cap S]}\left(u_{1}\right)=3$. Notice that $\left|N_{G[S]}(u) \cap N_{G[S]}\left(u_{1}\right)\right| \leqslant 2$ for each $u \in S \backslash\left\{u_{1}\right\}$ if $G\left[R_{4}\right]$ has a 4-cycle. It follows that $G\left[R_{4}\right]$ is isomorphic to $K_{1,4}$. Since $d_{G[\psi(S) \cap S]}(v)=2$ for each $v \in N_{G[\psi(S) \cap S]}\left(u_{1}\right)$, it follows that $N_{G[\psi(S) \cap S]}\left(u_{1}\right) \subseteq R_{3}$. It implies that the vertex in $R_{3} \backslash N_{G[S]}\left(u_{1}\right)$ has only two neighbors in $S$, which contradicts $\delta(G[S]) \geqslant 3$.

Lemma 26. Suppose $k=5, \lambda_{6}(G)=\lambda_{7}(G)=14$ and $g(G)>3$. For a $\lambda_{7}$-atom $S$ of $G$, we have $|S| \geqslant 14$.

Proof. By Lemma 23, we have $p=1,|X| \geqslant 10$ and $|V(H)| \geqslant 15$. Hence $|V(G)| \geqslant 32$. For $1 \leqslant i \leqslant 5$, let $O_{i}$ be the set of vertices $u$ in $G$ with $|\nabla(u) \cap(V(H))|=i$, and set $m_{i}=\left|O_{i} \cap X\right|$ and $n_{i}=\left|O_{i} \cap V(H)\right|$. By Lemma 20(a), $d_{G}(V(H) \cup A) \geqslant \lambda_{6}(G)$ and $d_{G}(V(H) \backslash A) \geqslant \lambda_{6}(G)$ for each subset $A$ of $V(G)$ with $|A| \leqslant 2$. This, together with the fact that $d_{G}(H)$ is odd, implies that $d_{G}(H)=15, O_{4} \cup O_{5}=\emptyset, m_{3} \leqslant 1$ and $n_{3} \leqslant 1$. Hence $E(X)=\emptyset$. Then $g_{0}(G) \geqslant 9$ by Lemma 17 .

Suppose $|S|<14$. As $5|S|-2|E(G[S])|=14,|S|$ is an even integer with $8 \leqslant|S| \leqslant 12$. By Lemma $20(\mathrm{~b}), \delta(G[S]) \geqslant 3$. As $g_{0}(G) \geqslant 9$, it follows that $G[S]$ is bipartite. By Lemma $20(\mathrm{a}), d_{G}(S \cup\{u\}) \geqslant \lambda_{6}(G)$ for each $u \in \bar{S}$ and $d_{G}(A) \geqslant \lambda_{6}(G)$ for each subset $A \subseteq V(G)$ with $|A|=6$. Hence $\left|N_{G}(u) \cap S\right| \leqslant 2$ for each $u \in \bar{S}$ and $G$ has no subgraphs which are isomorphic to $K_{3,3}$.

Claim 1. For any two distinct $\lambda_{7}$-atoms $S_{1}$ and $S_{2}$ of $G$ with $S_{1} \cap S_{2} \neq \emptyset$, we have $d_{G}\left(S_{1} \cap S_{2}\right) \leqslant 14$ and furthermore, $G\left[S_{1} \cap S_{2}\right]$ and $G\left[S_{1} \backslash S_{2}\right]$ are isomorphic to $K_{2,4}$ or $K_{3,3}-e$ if $|S|=12$, where $K_{3,3}-e$ is a subgraph of $K_{3,3}$ obtained by deleting an edge $e$ from $K_{3,3}$.

By Lemma 20(c), we have $\left|S_{1} \cap S_{2}\right| \leqslant 6,\left|S_{1} \backslash S_{2}\right| \leqslant 6, d_{G}\left(S_{1} \cap S_{2}\right)+d_{G}\left(S_{1} \cup S_{2}\right) \leqslant 2 \lambda_{7}(G)$ and $d_{G}\left(S_{1} \backslash S_{2}\right)+d_{G}\left(S_{2} \backslash S_{1}\right) \leqslant 2 \lambda_{7}(G)$. Noting $|V(G)| \geqslant 32$, we have $d_{G}\left(S_{1} \cup S_{2}\right) \geqslant \lambda_{7}(G)$ by Lemma $20(\mathrm{a})$. Hence $d_{G}\left(S_{1} \cap S_{2}\right) \leqslant \lambda_{7}(G)=14$. Next assume $|S|=12$. Then $\left|S_{1} \cap S_{2}\right|=\left|S_{1} \backslash S_{2}\right|=6$. By Lemma $20\left(\right.$ a), each of $d_{G}\left(S_{1} \cap S_{2}\right), d_{G}\left(S_{1} \backslash S_{2}\right)$ and $d_{G}\left(S_{2} \backslash S_{1}\right)$ is not less than $\lambda_{6}(G)$. Hence $d_{G}\left(S_{1} \cap S_{2}\right)=d_{G}\left(S_{1} \backslash S_{2}\right)=14$. It implies that $G\left[S_{1} \cap S_{2}\right]$ and $G\left[S_{1} \backslash S_{2}\right]$ are isomorphic to $K_{2,4}$ or $K_{3,3}-e$. So Claim 1 holds.

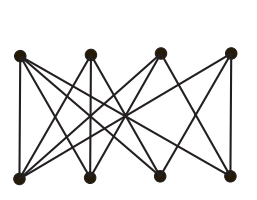

$G_{6}$

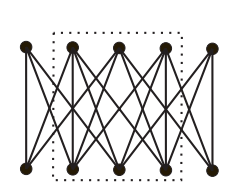

$G_{7}$

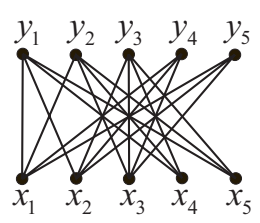

$G_{8}$

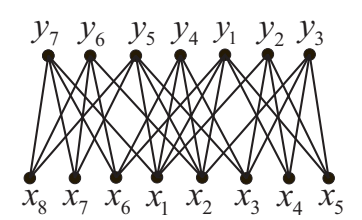

$G_{9}$

Figure 2. The illustration in the proof of Lemma 26.

Case 1. $|S|=8$. 
As $G[S]$ is a bipartite graph with $|E(S)|=13$ and $\delta(G[S]) \geqslant 3, G[S]$ is isomorphic to $G_{6}$ in Figure 2. Let $v_{1}, v_{2}$ be the two vertices in $S$ with $d_{G[S]}\left(v_{1}\right)=d_{G[S]}\left(v_{2}\right)=4$ and choose a vertex $v_{3} \in N_{G[S]}\left(v_{1}\right) \backslash\left\{v_{2}\right\}$.

We claim that each edge in $G$ is contained in a 4-cycle of $G$. Otherwise, suppose that $G$ has an edge contained in no 4-cycles of $G$. Since $G$ is vertex-transitive, each vertex in $G$ is incident with an edge contained in no 4-cycles of $G$ and there is an automorphism $\varphi_{1}$ of $G$ such that $\varphi_{1}\left(v_{3}\right)=v_{2}$. Clearly, $\varphi_{1}(S) \neq S$. Noting that each edge in $G[S]$ is contained in a 4-cycle of $G[S]$, we have $d_{G\left[\varphi_{1}(S) \cup S\right]}(u) \leqslant 4$ for each $u \in \varphi_{1}(S) \cup S$. Then $\varphi_{1}\left(N_{G[S]}\left(v_{3}\right) \subseteq N_{G[S]}\left(v_{2}\right)\right.$ and $N_{G[S]}\left(\varphi_{1}\left(v_{1}\right)\right) \subseteq \varphi_{1}\left(N_{G[S]}\left(v_{1}\right)\right)$. Noting that $\left|\varphi_{1}(S) \cap S\right| \leqslant 6$ by Lemma 20 (c) and $d_{G}\left(\varphi_{1}(S) \cap S\right) \leqslant 14$ by Claim $1, G\left[S \cap \varphi_{1}(S)\right]$ is isomorphic to $K_{3,3}-e$. As $d_{G}\left(S \cup \varphi_{1}(S)\right) \geqslant \lambda_{6}(G)=14$ by Lemma $20\left(\right.$ a), it follows that $G\left[S \cup \varphi_{1}(S)\right]$ is isomorphic to $G_{7}$ in Figure 2, where the graph in the virtual box corresponds to $G\left[S \cap \varphi_{1}(S)\right]$. Choose a vertex $v_{4} \in S \cap \varphi_{1}(S)$ with $d_{G\left[S \cap \varphi_{1}(S)\right]}\left(v_{4}\right)=2$. Let $\varphi_{2}$ be an automorphism of $G$ such that $\varphi_{2}\left(v_{1}\right)=v_{4}$. Then $\varphi_{2}\left(N_{G[S]}\left(v_{1}\right)\right)=N_{G\left[S \cup \varphi_{1}(S)\right]}\left(v_{4}\right)$ and $\varphi_{2}\left(N_{G[S]}\left(v_{2}\right)\right) \backslash\left(S \cup \varphi_{1}(S)\right) \neq \emptyset$. Then $d_{G}\left(S \cup \varphi_{2}(S) \cup \varphi_{2}\left(N_{G[S]}\left(v_{2}\right)\right)\right)<14=\lambda_{6}(G)$, contradicting Lemma 20(a). So this claim holds.

For each $u v \in \nabla(H)$, noting that $u v$ is contained in a 4-cycle of $G$ by the previous claim, we have $|\nabla(u) \cap \nabla(H)|+|\nabla(v) \cap \nabla(H)| \geqslant 3$. For each $u \in O_{2} \cup O_{3}$, there is an automorphism $\varphi_{3}$ of $G$ such that $\varphi_{3}\left(v_{1}\right)=u$, which implies that there is a vertex $v \in \varphi_{3}\left(N_{G[S]}\left(v_{1}\right)\right)$ such that $u v \in \nabla(H)$ and $|\nabla(v) \cap \nabla(H)|=3$. Hence $m_{1} \leqslant n_{2}+2 n_{3}$, $m_{2} \leqslant 3 n_{3}$ and $n_{2} \leqslant 3 m_{3}$. Noting $m_{3} \leqslant 1$ and $n_{3} \leqslant 1$, we have $15=\sum_{i=1}^{3} i m_{i} \leqslant$ $n_{2}+2 n_{3}+6 n_{3}+3 m_{3} \leqslant 6 m_{3}+8 n_{3} \leqslant 14$, a contradiction.

Case 2. $|S|=10$ or 12 .

Let $R_{i}$ be the set of vertices $u$ in $S$ with $d_{G[S]}(u)=i$ for $3 \leqslant i \leqslant 5$. Then $E\left(R_{3}\right)=\emptyset$ by Lemma 20(b). Let $Z$ and $W$ be the bipartition of $G[S]$ such that $|Z| \leqslant|W|$. Noting $\frac{1}{2}(5|S|-14)=|E(S)| \geqslant \delta(G[S])|W| \geqslant 3|W|$, we have $|W|<\frac{1}{2}|S|+2$.

Claim 2. If $R_{5} \neq \emptyset$, then, for each $v \in R_{4}$, there is exactly one vertex $w$ in $S \backslash\{v\}$ with $N_{G[S]}(v) \subseteq N_{G[S]}(w)$.

Suppose $R_{5} \neq \emptyset$. Choose a vertex $u \in R_{5}$ and a vertex $v \in R_{4}$. Let $\varphi_{4}$ be an automorphism of $G$ such that $\varphi_{4}(u)=v$. Then $N_{G[S]}(v) \subseteq \varphi_{4}\left(N_{G[S]}(u)\right)$. Noting that $\left|S \cap \varphi_{4}(S)\right| \leqslant 6$ by Lemma $20(\mathrm{c})$ and $d_{G}\left(S \cap \varphi_{4}(S)\right) \leqslant 14$ by Claim 1 , we have that $G\left[S \cap \varphi_{4}(S)\right]$ is isomorphic to $K_{2,4}$. It implies that $S$ has a vertex $w$ different from $v$ with $N_{G[S]}(v) \subseteq N_{G[S]}(w)$. As $G$ has no subgraphs isomorphic to $K_{3,3}$, such vertex $w$ is unique. So Claim 2 holds.

Claim 3. $|W|=|Z|$ and $R_{5}=\emptyset$.

Suppose, to the contrary, that $|W|>|Z|$, or $|W|=|Z|$ and $R_{5} \neq \emptyset$. As $E\left(R_{3}\right)=\emptyset$, it follows that $|W|=6$ if $|S|=10$.

Assume $|W|=|Z|+2=7$. Noting $|E(S)|=23$, there is a vertex $v_{5} \in\left(R_{4} \cup R_{5}\right) \cap W$ and a vertex $v_{6} \in R_{5} \cap Z$. Let $\varphi_{5}$ be an automorphism of $G$ such that $\varphi_{5}\left(v_{5}\right)=v_{6}$. Then $\varphi_{5}(S) \neq S$ and $\varphi_{5}\left(N_{G[S]}\left(v_{5}\right)\right) \subseteq N_{G[S]}\left(v_{6}\right)$. Hence $G\left[S \cap \varphi_{5}(S)\right]$ is isomorphic to $K_{2,4}$ by Claim 1. It implies $\left|\varphi_{5}(W) \backslash S\right|=5$, contradicting that $G\left[\varphi_{5}(S) \backslash S\right]$ is isomorphic to $K_{2,4}$ 
or $K_{3,3}-e$ by Claim 1 .

Assume $|W|=6$. If $|S|=10$, we know $\left|R_{4} \cap Z\right|=\left|R_{5} \cap Z\right|=2$ as $E\left(R_{3}\right)=\emptyset$ and $|E(S)|=18$. If $|S|=12$, we know either $\left|R_{5} \cap Z\right|=2=\left|R_{4} \cap Z\right|+1$ or $\left|R_{5} \cap Z\right|=1=$ $\left|R_{4} \cap Z\right|-2$ as $|E(S)|=23$. It follows from Claim 2 that there is a vertex $v_{7} \in R_{4} \cap Z$ and a vertex $v_{8} \in\left(R_{4} \cup R_{5}\right) \backslash\left\{v_{7}\right\}$ such that $N_{G[S]}\left(v_{7}\right) \subseteq N_{G[S]}\left(v_{8}\right)$ and $\left(R_{5} \cap Z\right) \backslash\left\{v_{8}\right\} \neq \emptyset$. It implies that $G[S]$ has a subgraph which is isomorphic to $K_{3,3}$, a contradiction. So Claim 3 holds.

Subcase 2.1. $|S|=10$.

Noting $E\left(R_{3}\right)=\emptyset$, we have by Claim 3 that $G[S]$ is isomorphic to $G_{8}$ in Figure 2. We label $G[S]$ as in $G_{8}$ and assume $x_{1} \in Z$ and $y_{1} \in W$.

Claim 4. $\left|N_{G}(u) \cap N_{G}(v)\right| \leqslant 3$ for any two distinct vertices $u$ and $v$ in $G$.

Suppose that there are two distinct vertices $u$ and $v$ in $G$ with $\left|N_{G}(u) \cap N_{G}(v)\right| \geqslant 4$. Notice that $\left|N_{G}(u) \cap S\right| \leqslant 2$ for each $u \in \bar{S}$. By the vertex-transitivity of $G$, for each $y_{i} \in\left\{y_{1}, y_{2}, y_{3}\right\}$ there is a vertex $y_{j} \in\left\{y_{1}, y_{2}, y_{3}\right\} \backslash\left\{y_{i}\right\}$ such that $\left|N_{G}\left(y_{i}\right) \cap N_{G}\left(y_{j}\right)\right| \geqslant 4$. It follows that there is a vertex $w \in \bar{S}$ such that $\left\{y_{1}, y_{2}, y_{3}\right\} \subseteq N_{G}(w)$, a contradiction. So Claim 4 holds.

Let $\varphi_{6}$ be an automorphism of $G$ such that $\varphi_{6}\left(y_{5}\right)=y_{1}$. Then $\varphi_{6}(S) \neq S$ and $\left|\varphi_{6}\left(N_{G[S]}\left(y_{5}\right)\right) \cap N_{G[S]}\left(y_{1}\right)\right| \geqslant 2$. Then $\left|\varphi_{6}(S) \cap S\right| \leqslant 6$ by Lemma $20\left(\right.$ c) and $d_{G}\left(\varphi_{6}(S) \cap\right.$ $S) \leqslant 14$ by Claim 1 . By Claim $4, G[S]$ has no subgraphs isomorphic to $K_{2,4}$. It follows that $\left|\varphi_{6}(S) \cap W\right| \leqslant 3$ and $\left|\varphi_{6}(S) \cap Z\right| \leqslant 3$.

Assume that $\varphi_{6}\left(N_{G[S]}\left(y_{5}\right)\right) \cap\left\{x_{1}, x_{2}\right\} \neq \emptyset$ and $\varphi_{6}\left(N_{G[S]}\left(y_{5}\right)\right) \cap\left\{x_{4}, x_{5}\right\} \neq \emptyset$. Then $\left|N_{G\left[\varphi_{6}(S)\right]}(u) \cap N_{G[S]}(u)\right|=3$ for each $u \in \varphi_{6}\left(N_{G[S]}\left(y_{5}\right)\right) \cap\left\{x_{1}, x_{2}\right\}$ and $\mid N_{G\left[\varphi_{6}(S)\right]}(v) \cap$ $N_{G[S]}(v) \mid \geqslant 2$ for each $v \in \varphi_{6}\left(N_{G[S]}\left(y_{5}\right)\right) \cap\left\{x_{4}, x_{5}\right\}$. It follows that $\left|\varphi_{6}(S) \cap W\right|=3$ and $\left|\varphi_{6}(S) \cap\left\{y_{4}, y_{5}\right\}\right|=1$. Noting $2 \leqslant\left|\varphi_{6}(S) \cap Z\right| \leqslant 3$, we can see $d_{G}\left(\varphi_{6}(S) \cap S\right)>14$, a contradiction.

Assume $\varphi_{6}\left(N_{G[S]}\left(y_{5}\right)\right) \cap N_{G[S]}\left(y_{1}\right)=\left\{x_{4}, x_{5}\right\}$. Then $\varphi_{6}\left(y_{4}\right) \in\left\{y_{2}, y_{3}\right\} \cup \bar{S}$, which implies that $\left|N_{G}\left(y_{1}\right) \cap N_{G}\left(\varphi_{6}\left(y_{4}\right)\right)\right| \geqslant 4$ or $\left|N_{G}\left(x_{4}\right) \cap N_{G}\left(x_{5}\right)\right| \geqslant 4$. It contradicts Claim 4.

Thus $\varphi_{6}\left(N_{G[S]}\left(y_{5}\right)\right) \cap N_{G[S]}\left(y_{1}\right)=\left\{x_{1}, x_{2}\right\}$. By Claim 4, we have $\varphi_{6}\left(y_{4}\right) \in\left\{y_{4}, y_{5}\right\}$ and $\varphi_{6}\left(\left\{y_{1}, y_{2}, y_{3}\right\}\right) \cap W=\left\{y_{4}, y_{5}\right\} \backslash \varphi_{6}\left(y_{4}\right)$. Then $\left\{\varphi_{6}\left(x_{4}\right), \varphi_{6}\left(x_{5}\right)\right\} \subseteq \bar{S}$. Assume $\varphi_{6}\left(y_{4}\right)=$ $y_{4}$. Set $\left\{y_{6}, y_{7}\right\}=\varphi_{6}\left(\left\{y_{1}, y_{2}, y_{3}\right\}\right) \backslash W,\left\{x_{6}\right\}=\varphi_{6}\left(N_{G[S]}\left(y_{5}\right)\right) \backslash N_{G[S]}\left(y_{1}\right)$ and $\left\{x_{7}, x_{8}\right\}=$ $\left\{\varphi_{6}\left(x_{4}\right), \varphi_{6}\left(x_{5}\right)\right\}$. Then the graph $G_{9}$ showed in Figure 2 is a subgraph of $G$.

We can see that each edge incident with $x_{1}$ is contained in a 4-cycle of $G$. Then, by the vertex-transitivity of $G$, each edge $u v \in \nabla(H)$ is contained in a 4-cycle of $G$, which implies $|\nabla(u) \cap \nabla(H)| \geqslant 2$ or $|\nabla(v) \cap \nabla(H)| \geqslant 2$. Hence there is a vertex $u^{\prime} \in G$ with $2 \leqslant\left|\nabla\left(u^{\prime}\right) \cap \nabla(H)\right| \leqslant 3$. Let $\varphi_{7}$ be an automorphism of $G$ such that $\varphi_{7}\left(y_{4}\right)=u^{\prime}$. It is easy to verify that either $\varphi_{7}\left(N_{G\left[\varphi_{6}(S) \cup S\right]}\left(y_{4}\right)\right)$ has a vertex $u$ with $|\nabla(u) \cap \nabla(H)| \geqslant 4$ or it has two vertices $v^{\prime}$ and $v^{\prime \prime}$ with $\left\{u^{\prime} v^{\prime}, u^{\prime} v^{\prime \prime}\right\} \subseteq \nabla(H)$ and $\left|\nabla\left(v^{\prime}\right) \cap \nabla(H)\right|=\left|\nabla\left(v^{\prime \prime}\right) \cap \nabla(H)\right|=3$, contradicting the fact that $O_{4} \cup O_{5}=\emptyset, m_{3} \leqslant 1$ and $n_{3} \leqslant 1$.

Subcase 2.2. $|S|=12$.

As $|E(G[S])|=23, G[S]$ is not regular. Let $\varphi_{8}$ be an automorphism of $G$ such that $\varphi_{8}(S) \neq S$ and $\varphi_{8}(S) \cap S \neq \emptyset$. Set $T=\varphi_{8}(S)$. It follows from Claims 1 and 3 that 
$d_{G[S \cup T]}(u)=5$ for each $u \in S \cap T$, each of $G[S \backslash T], G[S \cap T]$ and $G[T \backslash S]$ is isomorphic to $K_{3,3}-e$ and $d_{G[S]}(v)=d_{G[T]}(v)=4$ for each $v \in S \cap T$ with $d_{G[S \cap T]}(v)=3$.

Let $v_{9}$ and $v_{10}$ be two vertices in $W \cap T$ with $d_{G[S \cap T]}\left(v_{9}\right)=3=d_{G[S \cap T]}\left(v_{10}\right)+1$. We know either $d_{G[S]}\left(v_{10}\right)=4$ or $d_{G[T]}\left(v_{10}\right)=4$ and assume, without loss of generality, that $d_{G[S]}\left(v_{10}\right)=4$. Let $\varphi_{9}$ be an automorphism of $G$ such that $\varphi_{9}\left(v_{9}\right)=v_{10}$. Let $Q$ be one of $\varphi_{9}(S)$ and $\varphi_{9}(T)$ such that $Q \neq S$. Since $d_{G[Q]}\left(v_{10}\right)=4$, we know $Q \neq T$. By Claims 1 and 3, each of $G[Q \cap S], G[Q \backslash S], G[Q \cap T]$ and $G[Q \backslash T]$ is isomorphic to $K_{3,3}-e$. Noting $d_{G[S]}\left(v_{10}\right)=d_{G[Q]}\left(v_{10}\right)=4$, we have $\left|N_{G[Q]}\left(v_{10}\right) \cap S\right|=3$, which implies $2 \leqslant|Q \cap S \cap T| \leqslant 5$.

Assume $2 \leqslant|Q \cap S \cap T| \leqslant 3$. Noting that $G[Q \cap T]$ is isomorphic to $K_{3,3}-e$, we have $d_{G[Q \cap T]}(Q \cap S \cap T)>|Q \cap S \cap T| \geqslant d_{G[T]}(Q \cap S \cap T)$, a contradiction.

Assume $4 \leqslant|Q \cap S \cap T| \leqslant 5$. Then $\left|N_{G[Q]}\left(v_{10}\right) \cap S \cap T\right|=2$. If $E(Q \cap S \cap \bar{T})=\emptyset$, then $d_{G[Q \cap S]}(Q \cap S \cap \bar{T})+d_{G[Q \backslash T]}(Q \cap S \cap \bar{T}) \geqslant 4|Q \cap S \cap \bar{T}|>|[Q \cap S \cap \bar{T}, \bar{S} \cup T]|$, a contradiction. Thus $|Q \cap S \cap \bar{T}|=2$ and $|E(Q \cap S \cap \bar{T})|=1$. Then $d_{G[Q \cap S]}(Q \cap S \cap \bar{T})+d_{G[Q \backslash T]}(Q \cap S \cap \bar{T}) \geqslant$ $3+3>5 \geqslant|[Q \cap S \cap \bar{T}, \bar{S} \cup T]|$, a contradiction.

Lemma 27. Suppose $k=5, \lambda_{6}(G) \geqslant 14, \lambda_{8}(G)=15$ and $g(G)>3$. For a $\lambda_{8}$-atom $S$ of $G$, we have $|S| \geqslant 15$.

Proof. By Lemma 23, we have $p=1,|X| \geqslant 10$ and $|V(H)| \geqslant 15$. By Lemma 20(a), $d_{G}(A) \geqslant \lambda_{6}(G) \geqslant 14, d_{G}(V(H) \cup B) \geqslant \lambda_{8}(G)$ and $d_{G}(V(H) \backslash B) \geqslant \lambda_{8}(G)$ for any two subsets $A$ and $B$ of $V(G)$ with $|A|=6$ and $|B| \leqslant 1$. It implies that $G$ has no subgraphs isomorphic to $K_{3,3}, d_{G}(H)=15$ and $|\nabla(u) \cap \nabla(H)| \leqslant 2$ for each $u \in V(G)$. Hence $E(X)=\emptyset$ and there is an edge $u_{1} u_{2} \in \nabla(H)$ such that $N_{G}\left(u_{1}\right) \cap X=\left\{u_{2}\right\}$. By Lemma $17, g_{0}(G) \geqslant 9$.

Suppose $|S|<15$. As $g_{0}(G) \geqslant 9$ and $15=\lambda_{8}(G)=d_{G}(S)=5|S|-2|E(G[S])|$, it follows that $|S|$ is odd and $G[S]$ is bipartite. By Lemma 20(b), $\delta(G[S]) \geqslant 3$. Let $W$ and $Z$ be the bipartition of $G[S]$ such that $|W|>|Z|$. We have $|W|=\frac{1}{2}(|S|+1)$ if $|S| \leqslant 11$, and $7 \leqslant|W| \leqslant 8$ if $|S|=13$.

Case 1. There is a vertex $v_{1}$ in $S$ with $d_{G[S]}\left(v_{1}\right)=5$.

Let $R$ be one of $W$ and $Z$ such that $v_{1} \in R$. As $\delta(G[S]) \geqslant 3$ and $|E(S)|=\frac{1}{2}(5|S|-$ $2|E(G[S])|)$, it follows that $N_{G[S]}\left(N_{G[S]}\left(v_{1}\right)\right)=R$. Since $G$ is vertex-transitive, there is an automorphism $\varphi_{1}$ of $G$ such that $\varphi_{1}\left(v_{1}\right)=u_{2}$. Then $\varphi_{1}(R) \subseteq X \cup V(H)$. Noting that $|\nabla(u) \cap \nabla(H)| \leqslant 2$ for each $u \in V(G)$, we have $\varphi_{1}(S \backslash R) \cap X=\emptyset$. Notice that $G$ has no subgraphs isomorphic to $K_{3,3}$. We have $\left|\varphi_{1}(R) \cap X\right| \geqslant 4$ as $\left|N_{G}\left(u_{2}\right) \backslash V(H)\right| \geqslant 3$ and $\delta(G[S]) \geqslant 3$. Then $\left|\varphi_{1}(S) \cap V(H)\right| \leqslant 6$ as $|S| \leqslant 13$. It follows that $d_{G\left[\varphi_{1}(S)\right]}\left(u_{1}\right)=3$. Then $d_{G\left[\varphi_{1}(S)\right]}(v) \geqslant 4$ for each $v \in N_{G\left[\varphi_{1}(S)\right]}\left(u_{1}\right)$ by Lemma $20(\mathrm{~b})$. Now we know $|S|=13$, $\left|\varphi_{1}(R) \cap X\right|=4=\left|\varphi_{1}(R) \cap V(H)\right|+2$ and $\left|\varphi_{1}(S \backslash R) \cap V(H)\right|=4=\left|\varphi_{1}(S \backslash R) \backslash V(H)\right|+1$. Then $R=Z$ and $\left|N_{G}\left(u_{2}\right) \cap V(H)\right|=2$.

Noting that $|\nabla(u) \cap \nabla(H)| \leqslant 2$ for each $u \in V(G)$, we have $d_{G\left[\varphi_{1}(S)\right]}(u) \leqslant 4$ for each $u \in \varphi_{1}(W)$. Since $\delta(G[S]) \geqslant 3$ and $G$ has no subgraphs isomorphic to $K_{3,3}$, two vertices in $\varphi_{1}(W) \backslash V(H)$ has exactly 3 neighbors in $\varphi_{1}(Z) \cap X$. So $d_{G\left[\varphi_{1}(S)\right]}(u)=4$ for each $u \in \varphi_{1}(W) \backslash N_{G}\left(u_{2}\right)$ as $|E(S)|=25$. Then there is a vertex $u_{3} \in \varphi_{1}(Z) \cap X$ such that $\varphi_{1}(W) \backslash N_{G}\left(u_{2}\right) \subseteq N_{G}\left(u_{3}\right)$. 
Assume $\varphi_{1}(Z) \cap V(H)=\left\{u_{4}, u_{5}\right\}$. Let $\varphi_{2}$ be an automorphism of $G$ such that $\varphi_{2}\left(u_{4}\right)=$ $u_{2}$. Then $u_{1} \notin \varphi_{2}\left(N_{G\left[\varphi_{1}(S)\right]}\left(u_{4}\right)\right)$ and $\varphi_{2}\left(\left\{u_{2}, u_{3}, u_{5}\right\}\right) \subseteq X$, which implies $|\nabla(u) \cap \nabla(H)| \geqslant$ 3 for the vertex $u \in\left(N_{G}\left(u_{2}\right) \cap V(H)\right) \backslash\left\{u_{1}\right\}$, a contradiction.

Case 2. $d_{G[S]}(u) \leqslant 4$ for each $u \in S$.

If $|S|=13$, then, noting $|E(G[S])|=25$ and $5 \leqslant|Z| \leqslant 6$, we have that there is a vertex $u \in Z$ with $d_{G[S]}(u)=5$, a contradiction. Thus $|S| \leqslant 11$. There is a vertex $w \in W$ with $d_{G[S]}(w)=|W|-2$ such that $d_{G[S]}(u)=4$ for each $u \in N_{G[S]}(w)$. Choose a vertex $z \in N_{G[S]}(w)$.

We claim that the edge $u_{1} u_{2}$ is contained in a 4-cycle of $G$. Suppose not. Since $G$ is vertex-transitive, each vertex in $G$ is incident with an edge contained in no 4-cycles of $G$ and there is an automorphism $\varphi_{3}$ of $G$ such that $\varphi_{3}(w)=z$. We know $\varphi_{3}(S) \neq S$. Noting that $\left|N_{G[S]}(u) \cap N_{G[S]}(v)\right| \geqslant 2$ for every subset $\{u, v\} \subseteq Z$, each edge in $G[S]$ is contained in a 4-cycle of $G[S]$. Hence $\varphi_{3}\left(N_{G[S]}(w)\right) \subseteq N_{G[S]}(z)$ and $N_{G[S]}(u) \subseteq \varphi_{3}(S)$ for each $u \in \varphi_{3}\left(N_{G[S]}(w)\right)$. By Lemma $20(\mathrm{c}),\left|S \cap \varphi_{3}(S)\right| \leqslant 7$ and $d_{G}\left(S \cap \varphi_{3}(S)\right)+d_{G}\left(S \cup \varphi_{3}(S)\right) \leqslant$ $2 \lambda_{8}(G)$. If $|S|=11$, then $\left|S \cap \varphi_{3}(S)\right| \geqslant\left|\varphi_{3}\left(N_{G[S]}(w)\right) \cup N_{G[S]}(w)\right|=8$, a contradiction. Thus $|S|=9$. As $\delta(G[S]) \geqslant 3$, we have $Z=\bigcup_{u \in \varphi_{3}\left(N_{G[S]}(w)\right)} N_{G[S]}(u) \subseteq \varphi_{3}(S)$. Hence $\left|S \cap \varphi_{3}(S)\right|=7$ and $d_{G}\left(S \cap \varphi_{3}(S)\right)=17$. Noting that $d_{G}\left(S \cup \varphi_{3}(S)\right) \geqslant \lambda_{8}(G)$ by Lemma 20(a), we have $d_{G}\left(S \cap \varphi_{3}(S)\right)+d_{G}\left(S \cup \varphi_{3}(S)\right)>2 \lambda_{8}(G)$, a contradiction.

Thus $\left|N_{G}\left(u_{2}\right) \cap V(H)\right|=2$. Let $\varphi_{4}$ be an automorphism of $G$ such that $\varphi_{4}(z)=u_{2}$ if $|S|=9$, and $\varphi_{4}(w)=u_{2}$ if $|S|=11$. If $u_{1} \in \varphi_{4}(S)$, then $|Z| \geqslant d_{G\left[\varphi_{4}(S)\right]}\left(u_{1}\right)-$ $1+\left|N_{G\left[\varphi_{4}(S)\right]}\left(N_{G\left[\varphi_{4}(S)\right]}\left(u_{2}\right) \backslash V(H)\right)\right| \geqslant 2+3=5$ if $|S|=9$, and $|W| \geqslant 7$ if $|S|=11$, a contradiction. Thus $u_{1} \notin \varphi_{4}(S)$. Then $\varphi_{5}(Z) \subseteq X$ if $|S|=9$ and $\varphi_{5}(W) \subseteq X$ if $|S|=11$, which implies $|\nabla(u) \cap \nabla(H)| \geqslant 3$ for the vertex $u \in\left(N_{G}\left(u_{2}\right) \cap V(H)\right) \backslash\left\{u_{1}\right\}$, a contradiction.

Lemma 28. Suppose $k=6, \lambda_{5}(G)=16$ and $g(G)>3$. For a $\lambda_{5}$-atom $S$ of $G$, we have $|S| \geqslant 9$.

Proof. To the contrary, suppose $|S| \leqslant 8$. As $\frac{1}{2}\left(6|S|-\lambda_{5}(G)\right)=|E(S)| \leqslant \frac{1}{4}|S|^{2}$ by Lemma 6 , we have $|S| \geqslant 8$. Hence $|S|=8$ and $G[S]$ is isomorphic to $K_{4,4}$.

By Lemma $23, p=1$. Then $|X| \geqslant 7$ by Lemma 19 . Noting that $d(H) \leqslant 18$ and $H$ is triangle-free and factor-critical, we have $|V(H)| \geqslant 11$. Let $O_{i}$ be the set of vertices $u$ in $G$ with $|\nabla(u) \cap \nabla(H)|=i$ for $4 \leqslant i \leqslant 6$. By Lemma 20 (a), we have $d(V(H) \cup A) \geqslant \lambda_{5}(G)$ and $d(V(H) \backslash A) \geqslant \lambda_{5}(G)$ for each subset $A \subseteq V(G)$ with $|A| \leqslant 3$, which implies $d(H) \geqslant 16$, $O_{5} \cup O_{6}=\emptyset,\left|O_{4} \cap X\right| \leqslant 1$ and $\left|O_{4} \cap V(H)\right| \leqslant 1$.

Suppose that $S$ is an imprimitive block of $G$. Then the orbits $S=S_{1}, S_{2}, \ldots, S_{m}$ of $S$ under the automorphism group of $G$ form a partition of $V(G)$. If $E\left(S_{i}\right) \cap E(X) \neq \emptyset$ for some $S_{i}$, then $d(H)=16$ and $\left|S_{i} \cap V(H)\right|=6$, which implies $d\left(V(H) \cup S_{i}\right) \leqslant 14<\lambda_{5}(G)$, a contradiction. Thus $E\left(S_{j}\right) \cap E(X)=\emptyset$ for each $S_{j}$. As $c_{0}(G-X)=|X|-2$, it follows that $\left|O_{4}\right| \geqslant 3$, which contradicts the fact that $\left|O_{4}\right|=\left|O_{4} \cap X\right|+\left|O_{4} \cap V(H)\right| \leqslant 2$.

Suppose next that $S$ is not an imprimitive block of $G$. Then there is an automorphism $\varphi_{1}$ of $G$ such that $\varphi_{1}(S) \neq S$ and $\varphi_{1}(S) \cap S \neq \emptyset$. Set $T=\varphi_{1}(S)$. As $G$ is 6-regular, we have $\delta(G[S \cap T]) \geqslant 2$. By Lemma $20(\mathrm{c}),|S \cap T| \leqslant 4$. Hence $G[S \cap T]$ is a 4-cycle of $G$. Assume $S \cap T=\left\{v_{1}, v_{2}, v_{3}, v_{4}\right\}$, where $N\left(v_{1}\right)=N\left(v_{2}\right)$ and $N\left(v_{3}\right)=N\left(v_{4}\right)$. 
By the vertex-transitivity of $G$, for each $u \in V(G)$ there is a vertex $u^{\prime}$ different from $u$ such that $N\left(u^{\prime}\right)=N(u)$. Assume $E(X) \neq \emptyset$. Then $|E(X)|=1$ and let $u_{1} u_{2}$ be the edge in $E(X)$. We know that there is a vertex $u_{1}^{\prime}$ in $V(H)$ with $N\left(u_{1}^{\prime}\right)=N\left(u_{1}\right)$, which implies $\left|N\left(u_{1}\right) \cap V(H)\right|=5$. Then $O_{5} \neq \emptyset$, a contradiction. Thus $E(X)=\emptyset$. As for each $u \in V(G)$ there is a vertex $u^{\prime}$ different from $u$ such that $N\left(u^{\prime}\right)=N(u)$, it follows that there is a vertex $u_{3} \in X$ with $2 \leqslant\left|N\left(u_{3}\right) \cap V(H)\right| \leqslant 4$. Let $\varphi_{2}$ be an automorphism of $G$ such that $\varphi_{2}\left(v_{1}\right)=u_{3}$. If $\varphi_{2}\left(\left\{v_{3}, v_{4}\right\}\right) \backslash V(H) \neq \emptyset$, then $N\left(u_{3}\right) \cap V(H)=\varphi_{2}\left(N\left(v_{1}\right)\right) \cap V(H) \subseteq$ $\bigcup_{i=4}^{6} O_{i}$. If $\varphi_{2}\left(\left\{v_{3}, v_{4}\right\}\right) \subseteq V(H)$, then $\varphi_{2}\left(\left\{v_{3}, v_{4}\right\}\right) \subseteq \bigcup_{i=4}^{6} O_{i}$. So $\left|\left(\bigcup_{i=4}^{6} O_{i}\right) \cap V(H)\right| \geqslant 2$, a contradiction.

Lemma 29. Suppose $k=6, \lambda_{5}(G)=\lambda_{8}(G)=18$ and $g(G)>3$. For a $\lambda_{8}$-atom $S$ of $G$, we have $|S| \geqslant 15$.

Proof. To the contrary, suppose $8 \leqslant|S| \leqslant 14$. By Lemma 23 , we have $p=1,|X| \geqslant 7$ and $|V(H)| \geqslant 9$. By Lemma 20(a), we have $d_{G}(V(H) \cup A) \geqslant \lambda_{5}(G)$ and $d_{G}(V(H) \backslash A) \geqslant \lambda_{5}(G)$ for each subset $A \subseteq V(G)$ with $|A| \leqslant 1$, which implies $d_{G}(H)=18$ and $|\nabla(u) \cap \nabla(H)| \leqslant 3$ for each $u \in V(G)$. Then $g_{0}(G) \geqslant 7$ by Lemma 17. It follows that $G[A]$ is bipartite for each subset $A \subseteq V(G)$ with $|A| \leqslant 13$ and $d_{G}(A)=18$. Hence $|V(H)| \geqslant 15$, and $G[S]$ is bipartite if $|S| \leqslant 13$. Then $|V(G)| \geqslant 26$.

Case 1. $|S|=8$.

By Lemma $20(\mathrm{a}), d_{G}(A) \geqslant \lambda_{5}(G)$ for every subset $A \subseteq V(G)$ with $7 \leqslant|A| \leqslant 8$, which implies $\delta(G[S]) \geqslant 3$ and $G$ has no subgraphs isomorphic to $K_{4,4}$. As $|E(G[S])|=$ $\frac{1}{2}(6|S|-18)=15$ and $G[S]$ is bipartite, there is a vertex $u_{0} \in S$ with $d_{G[S]}\left(u_{0}\right)=3$ and $G\left[S \backslash\left\{u_{0}\right\}\right]$ is isomorphic to $K_{3,4}$.
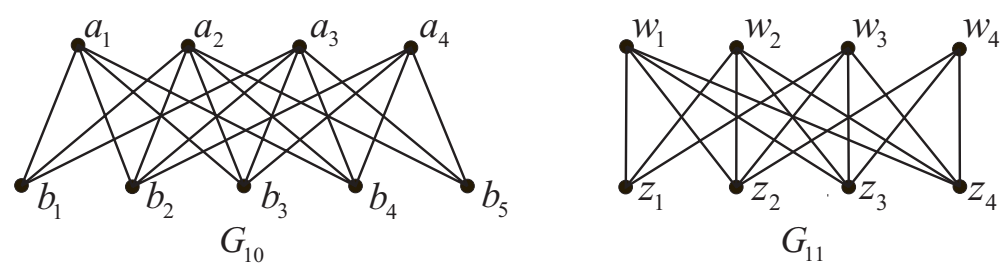

Figure 3. The illustration in the proof of Lemma 29.

Claim 1. There are no two distinct vertices $u$ and $v$ in $G$ with $N_{G}(u)=N_{G}(v)$.

Suppose that $u_{1}$ and $u_{2}$ are two distinct vertices in $G$ with $N_{G}\left(u_{1}\right)=N_{G}\left(u_{2}\right)$. Let $x, y$ and $z$ be the three vertices in $S$ which have 4 neighbors in $S \backslash\left\{u_{0}\right\}$. Noting that $G$ has no subgraphs isomorphic to $K_{4,4}$, we have, by the definition of the vertex-transitivity of $G$, that for each vertex $u \in\{x, y, z\}$ there is a vertex $u^{\prime} \in\{x, y, z\} \backslash\{u\}$ such that $N_{G}(u)=N_{G}\left(u^{\prime}\right)$. It follows that $N_{G}(x)=N_{G}(y)=N_{G}(z)$. Then $G$ is bipartite by Lemma 19, a contradiction. So Claim 1 holds.

Claim 2. G has no subgraphs isomorphic to $K_{3,5}$.

Suppose that $u_{3}, u_{4}$ and $u_{5}$ are three distinct vertices in $G$ with $\mid N_{G}\left(u_{3}\right) \cap N_{G}\left(u_{4}\right) \cap$ $N_{G}\left(u_{5}\right) \mid=5$. By Claim 1 and the vertex-transitivity of $G$, it follows that for each $u \in$ 
$N_{G}\left(u_{3}\right) \cap N_{G}\left(u_{4}\right)$ there are two distinct vertices $u^{\prime}, u^{\prime \prime} \in\left(N_{G}\left(u_{3}\right) \cap N_{G}\left(u_{4}\right)\right) \backslash\{u\}$ such that $\left|N_{G}(u) \cap N_{G}\left(u^{\prime}\right) \cap N_{G}\left(u^{\prime \prime}\right)\right|=5$. It implies that there is a vertex $v \in V(G) \backslash\left(\left\{u_{3}, u_{4}, u_{5}\right\}\right)$ such that $\left|N_{G}(v) \cap N_{G}\left(u_{3}\right) \cap N_{G}\left(u_{4}\right)\right| \geqslant 4$. So $G$ has a subgraph isomorphic to $K_{4,4}$, a contradiction. Claim 2 is proved.

Claim 3. $G$ has no subgraphs isomorphic to $G_{10}$ in Figure 3.

Suppose that $G_{10}$ is a subgraph of $G$. Let $\varphi_{1}$ be an automorphism of $G$ such that $\varphi_{1}\left(a_{2}\right)=a_{1}$. Noting that $d_{G}\left(V\left(G_{10}\right) \cup A\right) \geqslant \lambda_{5}(G)$ for each subset $A \subseteq V(G)$ with $|A| \leqslant 1$ by Lemma $20\left(\right.$ a), we have $G_{10}=G\left[V\left(G_{10}\right)\right]$ and $\left|N_{G}(u) \cap V\left(G_{10}\right)\right| \leqslant 3$ for each $u \in \overline{V\left(G_{10}\right)}$. We know $\varphi_{1}\left(a_{3}\right) \in\left\{a_{2}, a_{3}\right\}$ if $\left|\varphi_{1}\left(N_{G_{10}}\left(a_{2}\right)\right) \cap N_{G_{10}}\left(a_{1}\right)\right|=4$. Hence either each edge in $\nabla\left(a_{1}\right)$ or each edge in $\nabla\left(\varphi_{1}\left(a_{3}\right)\right)$ is contained in a 4-cycle of $G$. By the vertex-transitivity of $G$, each edge in $G$ is contained in a 4-cycle of $G$. It follows that $|\nabla(u) \cap \nabla(H)|+|\nabla(v) \cap \nabla(H)| \geqslant 3$ for each edge $u v \in \nabla(H)$.

We claim that $|\nabla(u) \cap \nabla(H)| \leqslant 2$ for each $u \in V(G)$. Otherwise, noting that $\mid \nabla(u) \cap$ $\nabla(V(H)) \mid \leqslant 3$ for each $u \in V(G)$, we suppose that there is a vertex $u_{6}$ in $G$ with $\mid \nabla\left(u_{6}\right) \cap$ $\nabla(H) \mid=3$. Let $\varphi_{2}$ be an automorphism of $G$ such that $\varphi_{2}\left(b_{2}\right)=u_{6}$. By considering the definition of $\varphi_{2}\left(V\left(G_{10}\right)\right)$, we can obtain that there is a vertex $u \in \varphi_{2}\left(N_{G_{10}}\left(b_{2}\right)\right)$ with $|\nabla(u) \cap \nabla(H)| \geqslant 4$, a contradiction.

Thus there a vertex $u_{7} \in V(G)$ with $\left|\nabla\left(u_{7}\right) \cap \nabla(H)\right|=2$. Let $\varphi_{3}$ be an automorphism of $G$ such that $\varphi_{3}\left(a_{2}\right)=u_{7}$. Then there is a vertex $u \in \varphi_{3}\left(N_{G_{10}}\left(a_{2}\right)\right)$ with $|\nabla(u) \cap \nabla(H)| \geqslant 3$, a contradiction. So Claim 3 holds.

By Claim 2, it follows that $G[S]$ is isomorphic to $G_{11}$ in Figure 3 and we label $G[S]$ as in $G_{11}$. Then $\left|N_{G}(u) \cap S\right| \leqslant 2$ for each $u \in \bar{S}$ by Claims 2 and 3. Let $\varphi_{4}$ be an automorphism of $G$ such that $\varphi_{4}\left(z_{1}\right)=z_{4}$. If $\varphi_{4}\left(N_{G[S]}\left(z_{1}\right)\right) \subseteq N_{G[S]}\left(z_{4}\right)$, then there is a vertex $u \in \varphi_{4}(S) \backslash S$ with $\left|N_{G}(u) \cap S\right| \geqslant 3$, a contradiction. Thus $\varphi_{4}\left(N_{G[S]}\left(z_{1}\right)\right) \backslash S \neq \emptyset$.

Assume $\varphi_{4}\left(N_{G[S]}\left(z_{1}\right)\right) \cap N_{G[S]}\left(z_{1}\right)=\left\{w_{i}, w_{j}\right\}$. As $\left|N_{G}(u) \cap S\right| \leqslant 2$ for each $u \in$ $\varphi_{4}\left(N_{G[S]}\left(z_{1}\right)\right) \backslash S$, it follows that $\left|\varphi_{4}\left(\left\{z_{2}, z_{3}, z_{4}\right\}\right) \backslash S\right|=2$. Then $N_{G}\left(w_{i}\right)=N_{G}\left(w_{j}\right)$, contradicting Claim 1.

Assume $\varphi_{4}\left(N_{G[S]}\left(z_{1}\right)\right) \cap N_{G[S]}\left(z_{1}\right)=\left\{w_{i^{\prime}}\right\}$. Then $\left|\varphi_{4}\left(\left\{z_{2}, z_{3}, z_{4}\right\}\right) \backslash S\right|=2$, which implies that each edge in $\nabla\left(w_{i^{\prime}}\right)$ is contained in a 4-cycle of $G$. Then each edge in $G$ is contained in a 4-cycle of $G$ by the vertex-transitivity of $G$. Thus there is a vertex $u_{8} \in V(G)$ with $2 \leqslant\left|\nabla\left(u_{8}\right) \cap \nabla(H)\right| \leqslant 3$. Let $\varphi_{5}$ be an automorphism of $G$ such that $\varphi_{5}\left(z_{4}\right)=u_{8}$. As $\left|N_{G}\left(w_{1}\right) \cap N_{G}\left(w_{2}\right) \cap N_{G}\left(w_{3}\right)\right|=4$ and $\left|N_{G}\left(\varphi_{4}\left(w_{1}\right)\right) \cap N_{G}\left(\varphi_{4}\left(w_{2}\right)\right) \cap N_{G}\left(\varphi_{4}\left(w_{3}\right)\right)\right|=4$, it follows that there is a vertex $u \in \varphi_{5}\left(N_{G\left[S \cup \varphi_{4}(S)\right]}\left(z_{4}\right)\right)$ with $|\nabla(u) \cap \nabla(H)| \geqslant 4$, a contradiction.

Thus $\varphi_{4}\left(N_{G[S]}\left(z_{1}\right)\right) \cap N_{G[S]}\left(z_{1}\right)=\emptyset$. By Claim 1, it follows that $\varphi_{4}\left(\left\{z_{2}, z_{3}, z_{4}\right\}\right)=$ $N_{G}\left(w_{4}\right) \backslash S$. Let $\varphi_{6}$ be an automorphism of $G$ such that $\varphi_{6}\left(z_{1}\right)=z_{3}$. Similarly, we have $\varphi_{6}\left(N_{G[S]}\left(z_{1}\right)\right) \cap N_{G[S]}\left(z_{1}\right)=\emptyset$ and $\varphi_{6}\left(\left\{z_{2}, z_{3}, z_{4}\right\}\right)=N_{G}\left(w_{4}\right) \backslash S$. It implies that $G\left[N_{G}\left(w_{4}\right) \cup \varphi_{4}\left(N_{G[S]}\left(z_{1}\right)\right) \cup \varphi_{6}\left(N_{G[S]}\left(z_{1}\right)\right)\right.$ has a subgraph isomorphic to $K_{3,5}$ or $G_{10}$, contradicting Claim 2 or Claim 3.

Case 2. $9 \leqslant|S| \leqslant 14$.

By Lemma $20(\mathrm{~b}), \delta(G[S]) \geqslant 4$. If $|S|=9$, then $18=\frac{1}{2}\left(6|S|-\lambda_{8}(G)\right)=|E(S)| \geqslant$ $\frac{1}{2}(|S|+1) \delta(G[S]) \geqslant 20$, a contradiction. Thus $|S| \geqslant 10$. If $|S| \leqslant 13$, then let $W$ and $Z$ 
be the bipartition of $G[S]$ with $|Z| \leqslant|W|$ and we have $|W|=|Z|+\frac{1}{2}\left(1-(-1)^{|S|}\right)$.

Subcase 2.1. $10 \leqslant|S| \leqslant 12$.

We claim that $d_{G[S]}(u) \leqslant 5$ for each $u \in S$. Otherwise, suppose that there is a vertex $v_{1} \in S$ with $d_{G[S]}\left(v_{1}\right)=6$. Choose a vertex $u_{9} \in X$ with $\nabla\left(u_{9}\right) \cap \nabla(H) \neq \emptyset$. Let $\varphi_{7}$ be an automorphism of $G$ such that $\varphi_{7}\left(v_{1}\right)=u_{9}$. As $\delta(G[S]) \geqslant 4$, it follows that $\varphi_{7}\left(S \backslash N_{G}\left(v_{1}\right)\right) \subseteq X$, which implies that $|\nabla(u) \cap \nabla(V(H))| \geqslant 4$ for each $u \in \varphi_{7}\left(N_{G}\left(v_{1}\right)\right) \cap$ $V(H)$, a contradiction.

Noting that $4 \leqslant d_{G[S]}(u) \leqslant 5$ for each $u \in S$, and recalling $|E(S)|=3|S|-9$ and $|W|=|Z|+\frac{1}{2}\left(1-(-1)^{|S|}\right)$, we know that there is a vertex $v_{2} \in Z$ and $v_{3} \in N_{G[S]}\left(v_{2}\right)$ such that $d_{G[S]}\left(v_{2}\right)=d_{G[S]}\left(v_{3}\right)+1=5$.

Now we claim that each edge in $G$ is contained in a 4-cycle of $G$. Otherwise, suppose that $G$ has an edge contained in no 4-cycles. By the definition of the vertex-transitivity of $G$, each vertex in $G$ is incident with an edge contained in no 4-cycles of $G$. Let $\varphi_{8}$ be an automorphism of $G$ such that $\varphi_{8}\left(v_{3}\right)=v_{2}$. Then $\varphi_{8}(S) \neq S$. Notice that each edge in $G[S]$ is contained in a 4-cycle of $G[S]$. We have $\varphi_{8}\left(N_{G[S]}\left(v_{3}\right)\right) \subseteq N_{G[S]}\left(v_{2}\right)$ and $N_{G[S]}\left(\varphi_{8}\left(v_{2}\right)\right) \subseteq \varphi_{8}\left(N_{G[S]}\left(v_{2}\right)\right)$. It implies $\left|\varphi_{8}(S) \cap S\right| \geqslant 8$, contradicting Lemma 20(c).

Thus $|\nabla(u) \cap \nabla(H)|+|\nabla(v) \cap \nabla(H)| \geqslant 3$ for each edge $u v \in \nabla(H)$. Then there is a vertex $u_{10} \in V(G)$ with $\left|\nabla\left(u_{10}\right) \cap \nabla(H)\right| \geqslant 2$.

Suppose $|S|=10$. Then $|W|=|Z|=5$. Let $\varphi_{9}$ be an automorphism of $G$ such that $\varphi_{9}\left(v_{2}\right)=u_{10}$. Then there is a vertex $u \in \varphi_{9}\left(N_{G[S]}\left(v_{2}\right)\right)$ with $|\nabla(u) \cap \nabla(H)| \geqslant 4$, a contradiction.

Thus $11 \leqslant|S| \leqslant 12$. Let $R_{i}$ be the set of vertices $u$ in $S$ with $d_{G[S]}(u)=i$ for $i=4,5$. Then $\left|R_{5}\right|=\left|R_{5} \cap Z\right|=4$ if $|S|=11$, and $\left|R_{5} \cap W\right|=\left|R_{5} \cap Z\right|=3$ if $|S|=12$.

Suppose that there is a vertex $u_{11} \in V(G)$ with $\left|\nabla\left(u_{11}\right) \cap \nabla(H)\right|=3$. For a vertex $v \in S$, let $\psi$ be an automorphism of $G$ such that $\psi(v)=u_{11}$. Then $\psi(S) \cap V(H) \neq \emptyset$ and $\psi(S) \backslash V(H) \neq \emptyset$. As $\delta(G[S]) \geqslant 4$ and $|\nabla(u) \cap \nabla(H)| \leqslant 3$ for each $u \in V(G)$, it follows that $|\psi(S) \cap X|=4$ and $G[\psi(S) \cap V(H)]$ and $G[\psi(S) \backslash V(H)]$ is isomorphic to $K_{1,4}$ or $K_{2,4}$. It implies $\left|N_{G[S]}(v) \cap R_{4}\right| \geqslant\left\lfloor\frac{|S|}{6}\right\rfloor$ and that there are two vertices $v^{\prime}, v^{\prime \prime} \in R_{4}$ with $N_{G[S]}\left(v^{\prime}\right)=N_{G[S]}\left(v^{\prime \prime}\right)$. If $|S|=11$, then $N_{G[S]}(u) \cap R_{4}=\emptyset$ for each $u \in W \backslash N_{G[S]}\left(R_{4} \cap Z\right)$, a contradiction. Thus $|S|=12$. Then $\left|N_{G[S]}(u) \cap R_{4}\right| \geqslant 2$ for each $u \in S$. So $\delta\left(G\left[R_{4}\right]\right) \geqslant 2$. Noting $\left|R_{4}\right|=\left|R_{5}\right|=6$, we have $12 \geqslant 4\left|R_{4}\right|-\delta\left(G\left[R_{4}\right]\right)\left|R_{4}\right| \geqslant 4\left|R_{4}\right|-2\left|E\left(R_{4}\right)\right|=$ $\left|\left[R_{4}, R_{5}\right]\right|=5\left|R_{5}\right|-2\left|E\left(R_{5}\right)\right| \geqslant 30-18$, which implies $d_{G\left[R_{4}\right]}(u)=2$ for each $u \in R_{4}$. Then $G\left[R_{4}\right]$ is a 6-cycle of $G$, which contradicts that $R_{4}$ has two vertices $v^{\prime}$ and $v^{\prime \prime}$ with $N_{G[S]}\left(v^{\prime}\right)=N_{G[S]}\left(v^{\prime \prime}\right)$.

So $|\nabla(u) \cap \nabla(H)| \leqslant 2$ for each $u \in V(G)$. Then $\left|\nabla\left(u_{10}\right) \cap \nabla(H)\right|=2$. We can see that there is no automorphism $\varphi$ of $G$ such that $\varphi\left(v_{2}\right)=u_{10}$, contradicting that $G$ is vertex-transitive.

Subcase 2.2. $13 \leqslant|S| \leqslant 14$.

Claim 4. For two distinct $\lambda_{8}$-atoms $S_{1}$ and $S_{2}$ of $G$ with $S_{1} \cap S_{2} \neq \emptyset, G\left[S_{1} \backslash S_{2}\right]$ and $G\left[S_{1} \cap S_{2}\right]$ are isomorphic to $K_{3,3}$ or $K_{3,4}$.

By Lemma $20(\mathrm{c})$, we have $\left|S_{1} \backslash S_{2}\right| \leqslant 7,\left|S_{1} \cap S_{2}\right| \leqslant 7, d_{G}\left(S_{1} \backslash S_{2}\right)+d_{G}\left(S_{2} \backslash S_{1}\right) \leqslant 2 \lambda_{8}(G)$ and $d_{G}\left(S_{1} \cap S_{2}\right)+d_{G}\left(S_{1} \cup S_{2}\right) \leqslant 2 \lambda_{8}(G)$. Then $\left|S_{1} \backslash S_{2}\right| \geqslant 6$ and $\left|S_{1} \cap S_{2}\right| \geqslant 6$. By Lemma 
20(a), each of $d_{G}\left(S_{1} \backslash S_{2}\right), d_{G}\left(S_{2} \backslash S_{1}\right), d_{G}\left(S_{1} \cap S_{2}\right)$ and $d_{G}\left(S_{1} \cup S_{2}\right)$ is not less than $\lambda_{5}(G)$. Noting $\lambda_{5}(G)=\lambda_{8}(G)=18$, we have $d_{G}\left(S_{1} \backslash S_{2}\right)=d_{G}\left(S_{1} \cap S_{2}\right)=18$. Hence $G\left[S_{1} \backslash S_{2}\right]$ and $G\left[S_{1} \cap S_{2}\right]$ are isomorphic to $K_{3,3}$ or $K_{3,4}$. So Claim 4 holds.

Since $G[S]$ is not a regular graph, there is an automorphism $\varphi_{10}$ of $G$ such that $\varphi_{10}(S) \neq S$ and $\varphi_{10}(S) \cap S \neq \emptyset$. Then $G\left[S \backslash \varphi_{10}(S)\right]$ and $G\left[S \cap \varphi_{10}(S)\right]$ are isomorphic to $K_{3,3}$ or $K_{3,4}$ by Claim 4 . Set $B=S \cap \varphi_{10}(S)$.

Claim 5. $S$ has no subset $A$ different from $S \backslash B$ and $B$ such that $G[A]$ is isomorphic to $K_{3,4}$ and $G[S \backslash A]$ is isomorphic to $K_{3,3}$ or $K_{3,4}$.

Suppose, to the contrary, that $S$ has a subset $A$ satisfying the above condition. Assume $|S|=13$. As $|W|=|Z|+1=7$, we know $|A \cap W|=4$. It follows that there is a vertex $v_{4} \in S$ with $d_{G[S]}\left(v_{4}\right)=6$. Choose a vertex $v_{5} \in S$ such that $d_{G[S]}\left(v_{5}\right) \geqslant 5$ and $\left|\left\{v_{4}, v_{5}\right\} \cap W\right|=1$. Let $\varphi_{11}$ be an automorphism of $G$ such that $\varphi_{11}\left(v_{5}\right)=v_{4}$. Then $\varphi_{11}(S) \neq S$ and $\varphi_{11}\left(N_{G[S]}\left(v_{5}\right)\right) \subseteq N_{G[S]}\left(v_{4}\right)$, contradicting that $G\left[S \cap \varphi_{11}(S)\right]$ is isomorphic to $K_{3,3}$ or $K_{3,4}$ by Claim 4 . Assume next $|S|=14$. Then each of $G[S \backslash B], G[B], G[A]$ and $G[S \backslash A]$ is isomorphic to $K_{3,4}$. As $|E(S)|=33$, we know $d_{G[S]}(B)=9$. If $|A \cap B|=1$, then $d_{G[S]}(B \backslash A)=9=\frac{1}{2} d_{G}(B \backslash A)$, contradicting Lemma 20 (b). If $|A \cap B|=6$, then $d_{G[S]}(S \backslash(A \cup B))=9=\frac{1}{2} d_{G}(S \backslash(A \cup B))$, contradicting Lemma 20(b). If $2 \leqslant|A \cap B| \leqslant 5$, then $9=d_{G[S]}(B) \geqslant d_{G[A]}(A \cap B)+d_{G[S \backslash A]}(B \backslash A) \geqslant 5+5$, a contradiction. Thus Claim 5 holds.

Claim 6. Each vertex in $G$ is contained in exactly two distinct $\lambda_{8}$-atoms of $G$.

By the vertex-transitivity of $G$, it only needs to show that $S^{\prime}=S$ or $\varphi_{10}(S)$ for a $\lambda_{8}$ atom $S^{\prime}$ of $G$ with $S^{\prime} \cap B \neq \emptyset$. Suppose $S^{\prime} \neq S$ and $S^{\prime} \neq \varphi_{10}(S)$. By Claims 4 and 5 , we have $S^{\prime} \cap S=B=S^{\prime} \cap \varphi_{10}(S)$. Then $18=d_{G}(B) \geqslant d_{G[S]}(B)+d_{G\left[\varphi_{10}(S)\right]}(B)+d_{G\left[S^{\prime}\right]}(B) \geqslant$ $3 \times 9$, a contradiction. Thus Claim 6 holds.

Let $D$ be one of $S \backslash B$ and $B$ such that $G[D]$ is isomorphic to $K_{3,4}$. Choose two vertices $v_{6}$ and $v_{7}$ in $D$ such that $d_{G[D]}\left(v_{6}\right)=d_{G[D]}\left(v_{7}\right)-1=3$. By Claim 6 , there is only one $\lambda_{8}$-atom $T$ of $G$ which is different from $S$ and contains $v_{6}$. By Claims 4 and 5 , we have $S \cap T=D$. By Claim 6,S and $T$ are also the only $\lambda_{8}$-atoms of $G$ which contain $v_{7}$. It implies that there is no automorphism $\varphi$ of $G$ such that $\varphi\left(v_{6}\right)=v_{7}$, a contradiction.

\section{Proof of Theorem 2}

In this section we complete the proof of Theorem 2.

Proof of Theorem 2. If $G$ is 4-factor-critical, then by Theorem 8 and Theorem 4 we have $k=\lambda(G) \geqslant 5$. So we consider the sufficiency. Suppose $k \geqslant 5$. We will prove that $G$ is 4 -factor-critical.

Suppose, to the contrary, that $G$ is not 4-factor-critical. We know by Theorem 1 that $G$ is bicritical. By Lemma 22, there is a subset $X \subseteq V(G)$ with $|X| \geqslant 4$ such that $c_{0}(G-X)=|X|-2$ and every component of $G-X$ is factor-critical. Let $H_{1}, H_{2}, \ldots$, $H_{p}, H_{p+1}, \ldots, H_{t}$ be the components of $G-X$, where $t=|X|-2$ and $H_{1}, H_{2}, \ldots, H_{p}$ 
are the nontrivial components of $G-X$. We know $p \geqslant 1$ by Lemma 23. For each $i \in[p]$, since $H_{i}$ is factor-critical, $\delta\left(H_{i}\right) \geqslant 2$. For every subset $J \subseteq[t]$, we have

$$
\sum_{i \in J} d_{G}\left(H_{i}\right)+\lambda(G)(t-|J|) \leqslant \sum_{i=1}^{t} d_{G}\left(H_{i}\right) \leqslant d_{G}(X)=k(t+2)-2|E(X)|,
$$

which implies

$$
\sum_{i \in J} d_{G}\left(H_{i}\right)+2|E(X)| \leqslant k(|J|+2)
$$

Hence $|E(X)| \leqslant k$. Set $Y=\bigcup_{j=p+1}^{t} V\left(H_{j}\right)$.

Case 1. $g(G)=3$.

By Lemma 18, $|E(X)| \geqslant t-p=|X|-2-p$.

Subcase 1.1. $d_{G}(A) \geqslant 2 k-2$ for all $A \subseteq V(G)$ with $2 \leqslant|A| \leqslant|V(G)|-2$.

For each $i \in[p]$, we have $d_{G}\left(H_{i}\right) \geqslant 2 k-2$. If $k$ is odd, then $d_{G}\left(H_{i}\right)$ is odd and hence $d_{G}\left(H_{i}\right) \geqslant 2 k-1$. So $d_{G}\left(H_{i}\right) \geqslant 2 k-\frac{1}{2}\left(3+(-1)^{k}\right)$ for each $i \in[p]$. Now we have

$$
\left(2 k-\frac{1}{2}\left(3+(-1)^{k}\right)\right) p+2(|X|-2-p) \leqslant \sum_{i=1}^{p} d_{G}\left(H_{i}\right)+2|E(X)| \leqslant k(p+2),
$$

which implies $\left(k-2-\frac{1}{2}\left(3+(-1)^{k}\right)\right) p+2(|X|-2-k) \leqslant 0$. Hence $|X| \leqslant k+1$.

Suppose $|X|<k$. Then $p=t=|X|-2$. By Theorem $9,|X| \geqslant \kappa(G)>\frac{2}{3} k$. Hence we know from $(2)$ that $2 k \geqslant\left(k-\frac{1}{2}\left(3+(-1)^{k}\right)\right) p>\left(k-\frac{1}{2}\left(3+(-1)^{k}\right)\right)\left(\frac{2}{3} k-2\right)$. That is, $k^{2}-7 k+3<0$ if $k$ is odd and $k^{2}-8 k+6<0$ otherwise. It follows that $k \leqslant 6$. If $k=6$, then $|X| \geqslant \kappa(G)=k$ by Lemma 11, a contradiction. Thus $k=5$. Then $\kappa(G)=|X|=4$. By Lemma $10, \tau(G)=2$. It implies that there is an edge $x_{0} y_{0} \in E(G)$ such that $\left|N_{G}\left(x_{0}\right) \cap N_{G}\left(y_{0}\right)\right|=4$.

Noting $k=5$, we know from (2) that $|E(X)| \leqslant 1$. Choose a vertex $u \in X$ with $d_{G[X]}(u)=0$. Since $G$ is vertex-transitive, there is an automorphism $\varphi_{1}$ of $G$ such that $\varphi_{1}\left(x_{0}\right)=u$. Assume, without loss of generality, that $\varphi_{1}\left(y_{0}\right) \in V\left(H_{1}\right)$. Noting $\mid N_{G}\left(x_{0}\right) \cap$ $N_{G}\left(y_{0}\right) \mid=4$, we have $N_{G}(u) \subseteq V\left(H_{1}\right)$. Then $d_{G}\left(V\left(H_{1}\right) \cup\{u\}\right)=d_{G}(X)-d_{G}\left(H_{2}\right)-5 \leqslant$ $20-9-5<2 k-2$, a contradiction.

Thus $k \leqslant|X| \leqslant k+1$. Noting $\left(k-2-\frac{1}{2}\left(3+(-1)^{k}\right)\right) p+2(|X|-2-k) \leqslant 0$, we have $p \leqslant 2$ and $k \leqslant 8$. Then $|Y|=|X|-2-p \geqslant k-4 \geqslant 1$. For any given vertex $v$, let $q$ be the number of triangles containing $v$ in $G$. By the vertex-transitivity of $G$, each vertex in $G$ is contained in $q$ triangles of $G$, which implies that each edge in $G$ is contained in at most $q$ triangles of $G$.

Claim 1. $E(X)$ is a matching of $G$.

Assume $p=2$ or $|X|=k+1$. Then we know from (2) that $|E(X)|=|X|-2-p=|Y|$. Since there are $q|Y|$ triangles of $G$ containing one vertex in $Y$, each edge in $E(X)$ is contained in $q$ triangles of $G$. It implies that $E(X)$ is a matching of $G$. Next we assume $p=1$ and $|X|=k$. If two edges in $E(X)$ are adjacent, then $|E(X)|=q \geqslant 2|Y|=2(k-3)$ 
and hence $d_{G}\left(H_{1}\right)+2|E(X)| \geqslant 2 k-2+4(k-3)>3 k$, which contradicts the inequality (1). So Claim 1 holds.

By Claim 1, it follows that each edge incident with a vertex in $Y$ is contained in at most one triangle of $G$. Then, by the vertex-transitivity of $G$, each edge in $E(X)$ is contained in at most one triangle of $G$.

Suppose $|X|=k+1$. From (2), we know $k \leqslant 6, p=1$ and $|E(X)|=|Y|=k-2$. Since $G$ has $q|Y|$ triangles containing one vertex in $Y$, each edge in $E(X)$ is contained in $q$ triangles of $G$. Noting that each edge in $E(X)$ is contained in at most one triangle of $G$, we have $q=1$. Then $\left|E\left(N_{G}(u)\right)\right|=1$ for each $u \in Y$, which implies $|X| \geqslant$ $2|E(X)|+(k-|E(X)|-1)=2 k-3>k+1$, a contradiction.

Thus $|X|=k$. Then for each $e \in E(X)$ and each $u \in Y, G$ has a triangle containing $e$ and $u$. As each edge in $E(X)$ is contained in at most one triangle of $G$, it follows that $|Y|=1$, which implies $p=2$ and $k=5$. From (2), we know $d_{G}\left(H_{1}\right)=d_{G}\left(H_{2}\right)=9$ and $|E(X)|=1$. Assume $\left|V\left(H_{1}\right)\right| \leqslant\left|V\left(H_{2}\right)\right|$. Let $u_{1}$ be the vertex in $Y$. For a vertex $u_{2} \in V\left(H_{1}\right)$ with $N_{G}\left(u_{2}\right) \cap X \neq \emptyset$, we have $\left|N_{G}\left(u_{2}\right) \cap X\right| \leqslant 3$ as $\delta\left(H_{1}\right) \geqslant 2$. As $H_{2}$ is a component of $G-N_{G}\left(u_{1}\right)$ with maximum cardinality, we have, by the definition of the vertex-transitivity of $G$, that $H_{2}$ also is a component of $G-N_{G}\left(u_{2}\right)$ with maximum cardinality. Then $N_{G}\left(X \backslash N_{G}\left(u_{2}\right)\right) \cap V\left(H_{2}\right)=\emptyset$. It implies $d_{G}\left(V\left(H_{1}\right) \cup\left(X \backslash N_{G}\left(u_{2}\right)\right)\right)<$ $8=2 k-2$, a contradiction. Hence Subcase 1.1 cannot occur.

Subcase 1.2. There is a subset $A \subseteq V(G)$ with $2 \leqslant|A| \leqslant|V(G)|-2$ such that $d_{G}(A)<2 k-2$.

We choose a subset $S$ of $V(G)$ such that $1<|S| \leqslant \frac{1}{2}|V(G)|, d(S)$ is as small as possible, and, subject to these conditions, $|S|$ is as small as possible. Then $d_{G}(S) \leqslant d_{G}(A) \leqslant 2 k-3$. By Corollary $14, d_{G}(S)=|S| \geqslant k$ and $G[S]$ is $(k-1)$-regular. As $2 k-3<\frac{2}{9}(k+1)^{2}, S$ is an imprimitive block of $G$ by Theorem 13. Thus $G[S]$ is vertex-transitive by Lemma 12 . We also know that the orbits $S=S_{1}, S_{2}, \ldots, S_{m_{1}}$ of $S$ under the automorphism group of $G$ form a partition of $V(G)$ and each $G\left[S_{i}\right]$ is $(k-1)$-regular.

Set $I_{i}=\left\{j \in\left\{1,2, \ldots, m_{1}\right\}: S_{j} \cap V\left(H_{i}\right) \neq \emptyset\right\}$ for each $i \in[t]$ and set $\mathscr{M}=\left\{\bigcup_{j \in I_{i}} S_{j}:\right.$ $i \in[t]\}$. If any two sets in $\mathscr{M}$ are disjoint, then $2|X| \geqslant 2\left|\bigcup_{U \in \mathscr{M}} \nabla(U)\right| \geqslant \sum_{U \in \mathscr{M}} d_{G}(U) \geqslant$ $|\mathscr{M}| d_{G}(S)$.

Suppose $|S|=k$. Then each $G\left[S_{i}\right]$ is isomorphic to $K_{k}$ and hence $G\left[S_{i}\right]$ has common vertices with at most one component of $G-X$. Hence $|\mathscr{M}|=c_{0}(G-X)=|X|-2$ and any two sets in $\mathscr{M}$ are disjoint. Then $2|X| \geqslant|\mathscr{M}| d_{G}(S)=(|X|-2) k>2|X|$, a contradiction.

Suppose $|S|=k+1$. As $\delta\left(H_{j}\right) \geqslant 2$ for each $j \in[p]$, we have that for each $S_{i}$, $\left|S_{i} \backslash X\right|=\left|S_{i} \cap Y\right|=2$ or $S_{i} \backslash X \subseteq V\left(H_{i^{\prime}}\right)$ for some $i^{\prime} \in[t]$. Hence $|\mathscr{M}| \geqslant p+\frac{1}{2}(t-$ $p)=\frac{1}{2}(t+p) \geqslant \frac{1}{2}(t+1)=\frac{1}{2}(|X|-1)$ and any two sets in $\mathscr{M}$ are disjoint. Then $2|X| \geqslant|\mathscr{M}| d_{G}(S) \geqslant \frac{1}{2}(|X|-1)(k+1)>2|X|$, a contradiction.

Thus $|S| \geqslant k+2$. Noting that $(k-1)|S|$ is even and $k+2 \leqslant|S| \leqslant 2 k-3$, we have $|S|=k+2$ if $5 \leqslant k \leqslant 6$. For each $i \in[p]$, if $V\left(H_{i}\right) \cap S_{j} \neq \emptyset$, then $\left|V\left(H_{i}\right) \cap S_{j}\right| \geqslant 2$ as $\delta\left(H_{i}\right) \geqslant 2$.

Claim 2. For each $S_{i}$, there is an element $a_{i} \in[p]$ such that $V\left(H_{a_{i}}\right) \cap S_{i} \neq \emptyset$. 
Suppose $S_{i} \subseteq X \cup Y$. By Lemma 16, $\left|S_{i} \cap Y\right| \leqslant \frac{1}{3}\left|S_{i}\right|$. If $k \geqslant 6$, then $|E(X)| \geqslant$ $\left|E\left(S_{i} \cap X\right)\right|=\frac{1}{2}(k-1)\left(\left|S_{i} \cap X\right|-\left|S_{i} \cap Y\right|\right) \geqslant \frac{1}{6}(k-1)\left|S_{i}\right| \geqslant \frac{1}{6}(k-1)(k+2)>k$, a contradiction. Thus $k=5$. Then $|S|=k+2$ and $\left|S_{i} \cap Y\right| \leqslant\left\lfloor\frac{1}{3}\left|S_{i}\right|\right\rfloor=2$. Hence $|E(X)| \geqslant\left|E\left(S_{i} \cap X\right)\right| \geqslant \frac{1}{2}(k-1)\left(\left|S_{i}\right|-4\right)=\frac{1}{2}(k-1)(k-2)>k$, a contradiction. So Claim 2 holds.

Claim 3. $X \backslash S_{i} \neq \emptyset$ for each $S_{i}$.

Suppose $X \subseteq S_{i}$. Choose a component $H_{j}$ of $G-X$ such that $H_{j} \neq H_{a_{i}}$. Then $\left|V\left(H_{j}\right) \cap S_{i}\right|=\left|N_{G}\left(V\left(H_{j}\right) \cap S_{i}\right) \backslash S_{i}\right| \leqslant\left|V\left(H_{j}\right) \backslash S_{i}\right|$. Hence $V\left(H_{j}\right) \backslash S_{i} \neq \emptyset$. Then there is some $S_{i^{\prime}} \subseteq V\left(H_{j}\right) \backslash S_{i}$. Now we know $d_{G}\left(V\left(H_{j}\right) \backslash S_{i}\right) \geqslant d_{G}(S)=\left|S_{i}\right|$. On the other hand, we have $d_{G}\left(V\left(H_{j}\right) \backslash S_{i}\right) \leqslant\left|S_{i} \backslash V\left(H_{a_{i}}\right)\right|<\left|S_{i}\right|$, a contradiction. So Claim 3 holds.

Claim 4. For each $i \in[p]$, we have $d_{G}\left(H_{i}\right) \geqslant 2 k-2$ if there is some $S_{j}$ such that $S_{j} \cap V\left(H_{i}\right) \neq \emptyset$ and $S_{j} \backslash V\left(H_{i}\right) \neq \emptyset$.

Suppose $S_{j} \cap V\left(H_{i}\right) \neq \emptyset$ and $S_{j} \backslash V\left(H_{i}\right) \neq \emptyset$. By Claim 3, $X \backslash S_{j} \neq \emptyset$. Suppose $\left|\overline{V\left(H_{i}\right) \cup S_{j}}\right|=1$. Then $\overline{V\left(H_{i}\right) \cup S_{j}}=X \backslash S_{j}$, which implies $\left|\overline{V\left(H_{i}\right) \cup X}\right|=1$. Hence $t=2$ and $p=1$, implying $t=|X|-2 \geqslant k-2>2$, a contradiction. Thus $\left|\overline{V\left(H_{i}\right) \cup S_{j}}\right| \geqslant 2$. Then $\left|S_{j}\right|=d_{G}(S) \leqslant d_{G}\left(V\left(H_{i}\right) \cup S_{j}\right) \leqslant\left|\left[V\left(H_{i}\right), \overline{V\left(H_{i}\right) \cup S_{j}}\right]\right|+\left|S_{j} \backslash V\left(H_{i}\right)\right|$, which implies $\left|\left[V\left(H_{i}\right), \overline{V\left(H_{i}\right) \cup S_{j}}\right]\right| \geqslant\left|S_{j} \cap V\left(H_{i}\right)\right|$. Hence $d_{G}\left(H_{i}\right) \geqslant d_{G\left[S_{j}\right]}\left(S_{j} \cap V\left(H_{i}\right)\right)+\mid\left[V\left(H_{i}\right), \overline{V\left(H_{i}\right)} \cap\right.$ $\left.\overline{S_{j}}\right]\left|\geqslant d_{G\left[S_{j}\right]}\left(S_{j} \cap V\left(H_{i}\right)\right)+\right| S_{j} \cap V\left(H_{i}\right) \mid$. If $\left|S_{j} \backslash V\left(H_{i}\right)\right| \geqslant 2$, then $d_{G\left[S_{j}\right]}\left(S_{j} \cap V\left(H_{i}\right)\right) \geqslant 2 k-4$ by Corollary 15 , which implies $d_{G}\left(H_{i}\right) \geqslant 2 k-4+\left|S_{j} \cap V\left(H_{i}\right)\right| \geqslant 2 k-2$. If $\left|S_{j} \backslash V\left(H_{i}\right)\right|=1$, then $d_{G}\left(H_{i}\right) \geqslant k-1+\left|S_{j} \cap V\left(H_{i}\right)\right| \geqslant 2 k$. Claim 4 holds.

Claim 5. $S_{i} \subseteq V\left(H_{a_{i}}\right) \cup X$ for each $S_{i}$.

Suppose, to the contrary, that $G-X$ has a component $H_{b}$ with $V\left(H_{b}\right) \cap\left(S_{i} \backslash V\left(H_{a_{i}}\right)\right) \neq$ $\emptyset$. Let $\theta$ be an integer such that $\theta=1$ if $\mid V\left(H_{b}\right)=1$ and $\theta=0$ otherwise. As $X \backslash S_{i} \neq \emptyset$ by Claim 2, there is some $S_{j}$ with $S_{j} \cap\left(X \backslash S_{i}\right) \neq \emptyset$. Set $J=\left\{a_{i}, b\right\} \cup\left\{a_{j}\right\}$. For each $i^{\prime} \in[p]$, we have $d_{G}\left(H_{i^{\prime}}\right) \geqslant d_{G}(S) \geqslant k+2$ and furthermore $d_{G}\left(H_{i^{\prime}}\right) \geqslant 2 k-2$ by Claim 4 if $i^{\prime} \in[p] \cap J$. If $|J|=2$, then, noting that $d_{G\left[S_{i}\right]}\left(V\left(H_{a_{j}}\right) \cap S_{i}\right) \geqslant 2 k-4$ by Corollary 15 and $\lambda\left(G\left[S_{j}\right]\right)=k-1$ by Theorem 8, we have $d_{G}\left(H_{a_{j}}\right) \geqslant d_{G\left[S_{i}\right]}\left(V\left(H_{a_{j}}\right) \cap S_{i}\right)+d_{G\left[S_{j}\right]}\left(V\left(H_{a_{j}}\right) \cap S_{j}\right) \geqslant 2 k-4+k-1=$ $3 k-5$.

Assume $5 \leqslant k \leqslant 6$. We know that $|S|=k+2$ and $G\left[S_{i} \cap V\left(H_{i^{\prime}}\right)\right]$ is isomorphic to $K_{2}$ for each $i^{\prime} \in\left\{a_{i}, b\right\} \cap[p]$. Hence $S_{i} \subseteq V\left(H_{a_{j}}\right) \cup V\left(H_{b}\right) \cup X$. If $\theta=1$, then $\left|E\left(G\left[S_{i} \cap X\right]\right)\right|=\frac{1}{2}\left((k-1)\left|S_{i} \cap X\right|-(k-1)-(2 k-4)\right)=\frac{1}{2}\left(k^{2}-5 k+6\right) \geqslant 3$. If $\theta=0$, then $k=6$ as $G\left[S_{i}\right]$ is vertex-transitive, which implies $\left|E\left(S_{i} \cap X\right)\right|=2$. Now we have

$$
\begin{aligned}
& \sum_{i^{\prime} \in J} d_{G}\left(H_{i^{\prime}}\right)+2|E(X)| \\
\geqslant & (3 k-5)(3-|J|)+2(2 k-2)(|J|-2)+\theta k+(1-\theta)(2 k-2)+2\left|E\left(S_{i} \cap X\right)\right| \\
= & k(|J|+2)+|J|+k-9-\theta(k-2)+2\left|E\left(S_{i} \cap X\right)\right|>k(|J|+2),
\end{aligned}
$$

which contradicts the inequality (1).

Assume $k \geqslant 7$. If $\theta=1$, then $t=|X|-2 \geqslant k-2 \geqslant 5$. If $\theta=0$, then $t=|X|-2 \geqslant$ 
$\left\lceil\frac{2 k}{3}\right\rceil-2 \geqslant 3$ by Theorem 9 . Now we have

$$
\begin{aligned}
& \sum_{i^{\prime} \in[t]} d_{G}\left(H_{i^{\prime}}\right)+2|E(X)| \\
\geqslant & (3 k-5)(3-|J|)+2(2 k-2)(|J|-2)+\theta(p-|J|+1)(k+2)+ \\
& (1-\theta)(2 k-2+(p-|J|)(k+2))+(t-p) k+2(t-p) \\
= & k(t+2)+2 t+\theta(k+2)+(1-\theta)(2 k-2)-|J|-k-7>k(t+2),
\end{aligned}
$$

which contradicts the inequality (1). So Claim 5 holds.

By Claims 2 and 5, it follows that $|\mathscr{M}|=p=t$ and any two sets in $\mathscr{M}$ are disjoint. Then $2|X| \geqslant|\mathscr{M}| d_{G}(S) \geqslant(|X|-2)(k+2)>2|X|$, a contradiction.

Case 2. $g(G) \geqslant 4$.

For each $j \in[p]$, we know from $(1)$ that $d_{G}\left(H_{j}\right) \leqslant 3 k$. Let $F_{j}$ be a component of $G\left[\overline{V\left(H_{j}\right)}\right]$ which contains a vertex in $V(G) \backslash\left(V\left(H_{j}\right) \cup X\right)$. Then $\nabla\left(F_{j}\right)$ is a 5 -restricted edge-cut of $G$. Hence $\lambda_{5}(G) \leqslant d_{G}\left(F_{j}\right) \leqslant d_{G}\left(H_{j}\right) \leqslant 3 k$. As it follows from Corollary 15 that $\lambda_{4}(G) \geqslant 2 k-2$, we have $2 k-2 \leqslant \lambda_{4}(G) \leqslant \lambda_{5}(G) \leqslant 3 k$.

Claim 6. If $\lambda_{5}(G) \geqslant 4 k-8$ and $k \leqslant 6$, then $p=1,\left|V\left(H_{1}\right)\right| \geqslant 7, \lambda_{7}(G) \leqslant 3 k$ and furthermore, $\lambda_{8}(G) \leqslant 3 k$ if $\lambda_{5}(G)>4 k-8$.

Suppose $\lambda_{5}(G) \geqslant 4 k-8$ and $k \leqslant 6$. Then $p=1$ by Lemma 23. We claim that $G\left[\overline{V\left(H_{1}\right)}\right]$ is connected. Otherwise, $d_{G}\left(H_{1}\right) \geqslant \lambda(G)+d_{G}\left(F_{1}\right) \geqslant k+\lambda_{5}(G)>3 k$, a contradiction. Suppose $\left|V\left(H_{1}\right)\right|=5$. As $g(G) \geqslant 4$ and $H_{1}$ is factor-critical, $H_{1}$ is a 5 -cycle of $G$. It follows that $k=5, E(X)=\emptyset$ and $|X| \geqslant 8$. Then $g_{0}(G) \geqslant 7$ by Lemma 17 , a contradiction. Thus $\left|V\left(H_{1}\right)\right| \geqslant 7$. Then $\nabla\left(H_{1}\right)$ is a 7-restricted edge-cut of $G$ and $\lambda_{7}(G) \leqslant d_{G}\left(V\left(H_{1}\right)\right) \leqslant 3 k$. If $\lambda_{5}(G)>4 k-8$, then $|X| \geqslant 7$ and $\left|V\left(H_{1}\right)\right| \geqslant 9$ by Lemma 23 , which implies $\lambda_{8}(G) \leqslant d_{G}\left(H_{1}\right) \leqslant 3 k$. So Claim 6 holds.

By Claim 6, we can discuss Case 2 in the following two subcases.

Subcase 2.1. $k=5, \lambda_{5}(G)=12$ and $\lambda_{7}(G) \geqslant 13$.

We have $\lambda_{4}(G)=12$. As $\lambda_{7}(G)$ exists, $|V(G)| \geqslant 14$. Then, by Lemma $20\left(\right.$ a),$d_{G}(A) \geqslant$ $\lambda_{7}(G)$ for each subset $A \subseteq V(G)$ with $|A|=7$, which implies that $G$ has no subgraphs isomorphic to $K_{3,4}$. By the definition of the vertex-transitivity of $G$, we can obtain that $G$ has no subgraphs isomorphic to $K_{2,5}$. By Claim $6, p=1$ and $\left|V\left(H_{1}\right)\right| \geqslant 7$. Hence $|X| \geqslant 6$ and $|V(G)| \geqslant 16$. By Lemma $20(\mathrm{a}), d_{G}\left(V\left(H_{1}\right) \cup A\right) \geqslant \lambda_{7}(G)$ for each subset $A \subseteq X$ with $|A| \leqslant 1$, which implies $d_{G}\left(H_{1}\right) \geqslant 13$ and $\left|N_{G}(u) \cap V\left(H_{1}\right)\right| \leqslant 3$ for each $u \in X$. Noting $\delta\left(H_{1}\right) \geqslant 2$, we have $\left|\nabla(u) \cap \nabla\left(H_{1}\right)\right| \leqslant 3$ for each $u \in V(G)$.

Claim 7. There is no subset $A \subseteq V(G)$ with $|A| \leqslant 3$ such that $A \cap V\left(H_{1}\right) \neq \emptyset$, $\left|\nabla(A) \cap \nabla\left(H_{1}\right)\right|=3|A|$ and $d_{G}\left(\left(V\left(H_{1}\right) \cup A\right) \backslash\left(V\left(H_{1}\right) \cap A\right)\right) \leqslant 12$.

Suppose, to the contrary, that such subset $A$ of $V(G)$ exists. Set $B=\left(V\left(H_{1}\right) \cup\right.$ $A) \backslash\left(V\left(H_{1}\right) \cap A\right)$. Then $|B| \geqslant 4$ and $|\bar{B}| \geqslant 7$. By Lemma 20(a), we have $d_{G}(B) \geqslant \lambda_{4}(G)$ and furthermore, $d_{G}(B) \geqslant \lambda_{7}(G)$ if $|B| \geqslant 7$. As $d_{G}(B) \leqslant 12$, we know $|B| \leqslant 6$ and $d_{G}(B)=12$. It implies that $E\left(V\left(H_{1}\right) \cap A\right)=\emptyset$ and $G[B]$ is isomorphic to $k_{2,2}$ or $K_{3,3}$. 
Hence $G\left[V\left(H_{1}\right) \cup A\right]$ is bipartite. Then $H_{1}$ is bipartite, contradicting the fact that $H_{1}$ is factor-critical. So Claim 7 holds.

As $\lambda_{5}(G)=12<\lambda_{7}(G)$ and $k=5$, each $\lambda_{5}$-atom of $G$ induces a subgraph which is isomorphic to $K_{3,3}$. Let $T_{1}, T_{2}, \ldots, T_{m_{2}}$ be all the subsets of $V(G)$, which induce subgraphs isomorphic to $K_{3,3}$. Let $R_{i}$ be the set of vertices in $X$ with $i$ neighbors in $V\left(H_{1}\right)$ for $1 \leqslant i \leqslant 3$ and let $Q$ be the set of vertices in $V\left(H_{1}\right)$ with 3 neighbors in $X$.

Subcase 2.1.1. There are two distinct $T_{i}$ and $T_{j}$ with $T_{i} \cap T_{j} \neq \emptyset$.

Noting that $G$ has no subgraphs isomorphic to $K_{3,4}$ or $K_{2,5}$, we have $\left|T_{i} \cap T_{j}\right|=2$ or 4. If $\left|T_{i} \cap T_{j}\right|=4$, then $d_{G}\left(T_{i} \cap T_{j}\right) \leqslant 12<\lambda_{7}(G)$, which contradicts Lemma 20(a). Thus $\left|T_{i} \cap T_{j}\right|=2$. Assume $T_{i} \cap T_{j}=\left\{v_{1}, v_{2}\right\}$.

Claim 8. For each $u \in X$ with $d_{G[X]}(u)=0$ and $N_{G}(u) \cap V\left(H_{1}\right) \neq \emptyset$, we have $N_{G}(u) \cap V\left(H_{1}\right) \subseteq Q$ if $u \in R_{1} \cup R_{2}$, and $\left|N_{G}(u) \cap V\left(H_{1}\right) \cap Q\right| \geqslant 1$ if $u \in R_{3}$.

Since $G$ is vertex-transitive, there is an automorphism $\varphi_{2}$ of $G$ such that $\varphi_{2}\left(v_{1}\right)=u$. If $u \in R_{1} \cup R_{2}$, then $\varphi_{2}\left(N_{G}\left(v_{2}\right)\right) \subseteq X$, which implies $N_{G}(u) \cap V\left(H_{1}\right) \subseteq Q$. If $u \in R_{3}$, then $\left|\varphi_{2}\left(N_{G}\left(v_{2}\right)\right) \cap X\right| \geqslant 3$, which implies $\left|N_{G}(u) \cap V\left(H_{1}\right) \cap Q\right| \geqslant 1$. So Claim 8 holds.

Assume $E(X) \neq \emptyset$. Then $|E(X)|=1$ and $\sum_{i=1}^{3} i\left|R_{i}\right|=d_{G}\left(H_{1}\right)=13$, which implies $\sum_{i=1}^{3}\left|R_{i}\right| \geqslant 5$. By Claim 8, $Q \neq \emptyset$. We have $d_{G}\left(V\left(H_{1}\right) \backslash\{u\}\right) \leqslant 12$ for each $u \in Q$, contradicting Claim 7.

Thus $E(X)=\emptyset$. As $d_{G}\left(V\left(H_{1}\right) \cup A\right) \geqslant \lambda_{4}(G)$ for each subset $A \subseteq X$ with $|A|=4$ by Lemma 20(a), we have $\left|R_{3}\right| \leqslant 3$. By Claim 8, $\left|\nabla(Q) \cap \nabla\left(H_{1}\right)\right| \geqslant\left|R_{3}\right|+2\left|R_{2}\right|+\left|R_{1}\right|=$ $15-2\left|R_{3}\right| \geqslant 9$, which implies $|Q| \geqslant 3$. Choose a subset $Q^{\prime} \subseteq Q$ with $\left|Q^{\prime}\right|=3$. Then $d_{G}\left(V\left(H_{1}\right) \backslash Q^{\prime}\right) \leqslant 12$, contradicting Claim 7. Hence Subcase 2.1.1 cannot occur.

Subcase 2.1.2. Any two distinct $T_{i}$ and $T_{j}$ are disjoint.

By the vertex-transitivity of $G$, each vertex in $G$ is contained in a $\lambda_{5}$-atom of $G$. Hence $T_{1}, T_{2}, \ldots, T_{m_{2}}$ form a partition of $V(G)$.

Assume $E(X) \neq \emptyset$. As $c_{0}(G-X)=|X|-2$ and $|E(X)|=1$, it follows that there is some $T_{i}$ such that $T_{i} \cap X \neq \emptyset, T_{i} \cap V\left(H_{1}\right) \neq \emptyset$ and $E\left(T_{i}\right) \cap E(X)=\emptyset$. Then there is a vertex $u_{1} \in T_{i} \cap\left(R_{3} \cup Q\right)$. By Claim 7, it follows that $u_{1} \in X$. We have $d_{G}\left(V\left(H_{1}\right) \cup\left\{u_{1}\right\}\right)=$ $12<\lambda_{7}(G)$, contradicting Lemma 20(a).

Thus $E(X)=\emptyset$. Set $\mathscr{B}_{1}=\left\{T_{j}:\left|T_{j} \cap X\right|=3, j \in\left[m_{2}\right]\right\}$ and $\mathscr{B}_{2}=\left\{T_{j}:\left|T_{j} \cap X\right|<\right.$ $\left.3, j \in\left[m_{2}\right]\right\}$. Let $D=\left(\bigcup_{A \in \mathscr{B}_{1}} A \cap V\left(H_{1}\right)\right) \cup\left(\bigcup_{A \in \mathscr{B}_{2}} A \cap X\right)$. Noting $c_{0}(G-X)=$ $|X|-2$ and $p=1$, we have $|D|=3$. By Claim 7 , we have $D \subseteq X$. If $|X| \geqslant 7$, then $d_{G}\left(H_{1}+D\right)=12<\lambda_{7}(G)$, which contradicts Lemma 20(a). Thus $|X|=6$. As $G$ has no subgraphs isomorphic to $K_{2,5}$, we know that $\left|R_{2}\right|=\left|R_{3}\right|=3$ and $G\left[Y \cup R_{2}\right]$ is isomorphic to $K_{3,3}$. Choose a vertex $u_{3} \in R_{3}$ and a vertex $u_{4} \in Y$. Let $\varphi_{3}$ be an automorphism of $G$ such that $\varphi_{3}\left(u_{4}\right)=u_{3}$. Noting $\varphi_{3}\left(Y \cup R_{2}\right) \cap\left(Y \cup R_{2}\right)=\emptyset$, we have $\varphi_{3}(Y)=R_{3}$ and $\varphi_{3}\left(R_{2}\right) \subseteq V\left(H_{1}\right)$. It implies $D \subseteq V\left(H_{1}\right)$ by the choice of $D$, a contradiction. Hence Subcase 2.1 cannot occur.

Subcase 2.2. $k \neq 5, \lambda_{5}(G) \neq 12$ or $\lambda_{5}(G)=\lambda_{7}(G)=12$. 
Let $S^{\prime}$ be a $\lambda_{s}$-atom of $G$, where

$$
s=\left\{\begin{array}{l}
4, \text { if } k \leqslant 6 \text { and } \lambda_{5}(G)<4 k-8 \\
7, \text { if } k=5 \text { and } \lambda_{5}(G)=\lambda_{7}(G)=12 ; \\
6, \text { if } k=5 \text { and } \lambda_{5}(G)=\lambda_{6}(G)=13 \\
7, \text { if } k=5, \lambda_{5}(G)=13 \text { and } \lambda_{6}(G)=\lambda_{7}(G)=14 ; \\
8, \text { if } k=5, \lambda_{5}(G)=13, \lambda_{6}(G) \geqslant 14 \text { and } \lambda_{8}(G)=15 ; \\
5, \text { if } k=5 \text { and } \lambda_{5}(G)=14 \\
6, \text { if } k=5 \text { and } \lambda_{5}(G)=\lambda_{6}(G)=15 ; \\
5, \text { if } k=6 \text { and } \lambda_{5}(G)=4 k-8 ; \\
8, \text { if } k=6 \text { and } \lambda_{5}(G)=18 \\
5, \text { if } k \geqslant 7 .
\end{array}\right.
$$

Claim 9. $S^{\prime}$ is an imprimitive block of $G$ such that $\left|S^{\prime}\right|>\frac{1}{2} \lambda_{s}(G)$ if $k \leqslant 6$ and $\left|S^{\prime}\right|>\frac{1}{3} \lambda_{s}(G)$ otherwise.

If $k=5$ and $\lambda_{5}(G)=\lambda_{7}(G)=12$, then, by Lemma 24, Claim 9 holds. So we assume $k>5$ or $\lambda_{5}(G) \neq 12$. By Lemma $6, \frac{1}{2}\left|S^{\prime}\right|^{2} \geqslant 2\left|E\left(S^{\prime}\right)\right|=k|S|-\lambda_{s}(G)$. If $5 \leqslant k \leqslant 6$ and $\lambda_{5}(G)<4 k-8$, then $\frac{1}{2}\left|S^{\prime}\right|^{2} \geqslant k\left|S^{\prime}\right|-\lambda_{s}(G)>k\left|S^{\prime}\right|-4 k+8$, which implies $\left|S^{\prime}\right|>$ $2 k-4 \geqslant \max \left\{2(s-1), \frac{1}{2} \lambda_{s}(G)\right\}$. If $5 \leqslant k \leqslant 6$ and $\lambda_{5}(G) \geqslant 4 k-8$, then $\left|S^{\prime}\right|>2(s-1)$ and $2\left|S^{\prime}\right|>\lambda_{s}(G)$ by Lemmas 21 and $25-29$. If $k \geqslant 7$, then $\frac{1}{2}\left|S^{\prime}\right|^{2} \geqslant k\left|S^{\prime}\right|-\lambda_{s}(G) \geqslant k\left|S^{\prime}\right|-3 k$ and hence $\left|S^{\prime}\right|>k+2>\max \left\{2(s-1), \frac{1}{3} \lambda_{s}(G)\right\}$. Suppose $S^{\prime}$ is not an imprimitive block of $G$. Then there is an automorphism $\varphi$ of $G$ such that $\varphi\left(S^{\prime}\right) \neq S^{\prime}$ and $\varphi\left(S^{\prime}\right) \cap S^{\prime} \neq \emptyset$. By Lemma 20(c), $\left|S^{\prime}\right|=\left|S^{\prime} \cap \varphi\left(S^{\prime}\right)\right|+\left|S^{\prime} \backslash \varphi\left(S^{\prime}\right)\right| \leqslant 2(s-1)$, a contradiction. So Claim 9 holds.

By Claim 9 and Lemma 12, $G\left[S^{\prime}\right]$ is vertex-transitive and hence it is (k-1)-regular if $k \leqslant 6$ and is $(k-1)$-regular or $(k-2)$-regular otherwise. From Claim 9 , we also know that the orbits $S^{\prime}=S_{1}^{\prime}, S_{2}^{\prime}, \ldots, S_{m_{3}}^{\prime}$ of $S^{\prime}$ under the automorphism group of $G$ form a partition of $V(G)$.

Claim 10. $G\left[S^{\prime}\right]$ is $(k-1)$-regular.

Suppose that $G\left[S^{\prime}\right]$ is $(k-2)$-regular. Then $k \geqslant 7, s=5$ and $2\left|S^{\prime}\right|=\lambda_{s}(G) \leqslant 3 k$, which implies $\left|S^{\prime}\right| \leqslant \frac{3}{2} k$. By Lemma $6, \frac{1}{4}\left|S^{\prime}\right|^{2} \geqslant\left|E\left(S^{\prime}\right)\right|=\frac{1}{2}(k-2)\left|S^{\prime}\right|$, which implies $\left|S^{\prime}\right| \geqslant 2(k-2)$. Now $2(k-2) \leqslant\left|S^{\prime}\right| \leqslant \frac{3}{2} k$, which implies $k \leqslant 8$ and $\left|S^{\prime}\right|=2(k-2)$. Hence $G\left[S^{\prime}\right]$ is isomorphic to $K_{k-2, k-2}$. For each $i \in[p]$, noting $3 k \geqslant d_{G}\left(H_{i}\right) \geqslant \lambda_{s}(G)=4(k-2)$ and that $d_{G}\left(H_{i}\right)$ has the same parity with $k$, we have $d_{G}\left(H_{i}\right)=3 k$. Hence $p=1$, $E(X)=\emptyset,\left|V\left(H_{1}\right)\right|>5$ and $|X| \geqslant k$. As $c_{0}(G-X)=|X|-2$, there is some $S_{i}^{\prime}$ with $S_{i}^{\prime} \cap X \neq \emptyset$ and $S_{i}^{\prime} \cap V\left(H_{1}\right) \neq \emptyset$. Then there is a vertex $u \in S_{i}^{\prime}$ with $\left|\nabla(u) \cap \nabla\left(H_{1}\right)\right| \geqslant k-2$. Then $d_{G}\left(V\left(H_{1}\right) \cup\{u\}\right) \leqslant d_{G}\left(H_{1}\right)-(k-4)=2 k+4<4(k-2)=\lambda_{s}(G)$ if $u \in X$ and $d_{G}\left(V\left(H_{1}\right) \backslash\{u\}\right)<\lambda_{s}(G)$ otherwise, contradicting Lemma 20(a). So Claim 10 holds.

As $\delta\left(H_{i}\right) \geqslant 2$ for each $i \in[p]$, it follows from Claim 10 that $\delta\left(G\left[V\left(H_{j}\right) \cap S_{i}^{\prime}\right]\right) \geqslant 1$ if $V\left(H_{j}\right) \cap S_{i}^{\prime} \neq \emptyset$. 
Claim 11. For each $S_{i}^{\prime}, S_{i}^{\prime} \backslash(X \cup Y) \neq \emptyset$ or $\left|S_{i}^{\prime} \cap X\right|=\left|S_{i}^{\prime} \cap Y\right|$.

Suppose $\left|S_{i}^{\prime} \cap X\right|>\left|S_{i}^{\prime} \cap Y\right|$ for some $S_{i}^{\prime} \subseteq X \cup Y$. If $G\left[S_{i}^{\prime}\right]$ is bipartite, then $\left|S_{i}^{\prime} \cap Y\right| \leqslant$ $\left|S_{i}^{\prime} \cap X\right|-2$. If $G\left[S_{i}^{\prime}\right]$ is non-bipartite, then $\left|S_{i}^{\prime} \cap Y\right| \leqslant \alpha\left(G\left[S_{i}^{\prime}\right]\right) \leqslant \frac{1}{2}\left|S_{i}^{\prime}\right|-\frac{k-1}{4}$ by Lemma 16, which implies $\left|S_{i}^{\prime} \cap Y\right| \leqslant\left|S_{i}^{\prime} \cap X\right|-\frac{k-1}{2} \leqslant\left|S_{i} \cap X\right|-2$. Thus $\left.\mid E\left(S_{i}^{\prime} \cap X\right]\right) \mid=$ $\frac{1}{2}(k-1)\left(\left|S_{i}^{\prime} \cap X\right|-\left|S_{i}^{\prime} \cap Y\right|\right) \geqslant k-1$. Noting $d_{G}\left(H_{1}\right) \geqslant \lambda_{5}(G) \geqslant 2 k-2$, we have $d_{G}\left(H_{1}\right)+2|E(X)| \geqslant 2 k-2+2(k-1)>3 k$, a contradiction. So Claim 11 holds.

Subcase 2.2.1. $\left|S^{\prime}\right| \leqslant 2 k-1$.

Claim 12. If $S_{i}^{\prime} \cap V\left(H_{j}\right) \neq \emptyset$ for some $j \in[p]$, then $S_{i}^{\prime} \subseteq V\left(H_{j}\right) \cup X$.

Suppose $S_{i}^{\prime} \cap V\left(H_{j}\right) \neq \emptyset$ for some $j \in[p]$ and $S_{i}^{\prime} \cap V\left(H_{j^{\prime}}\right) \neq \emptyset$ for some $j^{\prime} \in[t] \backslash\{j\}$. As $\delta\left(G\left[S_{i}^{\prime} \cap V\left(H_{j}\right)\right]\right) \geqslant 1$, there is an edge $x_{1} y_{1} \in E\left(S_{i}^{\prime} \cap V\left(H_{j}\right)\right)$. Then $\left|S_{i}^{\prime} \cap\left(V\left(H_{j}\right) \cup X\right)\right| \geqslant$ $\left|N_{G\left[S_{i}^{\prime}\right]}\left(x_{1}\right) \cup N_{G\left[S_{i}^{\prime}\right]}\left(y_{1}\right)\right|=2 k-2$. It implies $\left|S_{i}^{\prime} \cap V\left(H_{j^{\prime}}\right)\right|=1$ and $\left|S_{i}^{\prime}\right|=2 k-1$. Then $\left|V\left(H_{j^{\prime}}\right)\right|=1$ and $|X| \geqslant\left|N_{G}\left(V\left(H_{j^{\prime}}\right)\right)\right|=k$. Hence $\left|\overline{V\left(H_{j}\right) \cup S_{i}^{\prime}}\right| \geqslant\left|N_{G}\left(V\left(H_{j^{\prime}}\right)\right) \backslash S_{i}^{\prime}\right|+$ $\left(c_{0}(G-X)-2\right) \geqslant 1+k-4 \geqslant 2$. By Corollary 15 , we have

$$
\begin{aligned}
2 k-2 & \leqslant d_{G}\left(V\left(H_{j}\right) \cup S_{i}^{\prime}\right) \\
& \leqslant d_{G}\left(H_{j}\right)-d_{G\left[S_{i}^{\prime}\right]}\left(S_{i}^{\prime} \cap V\left(H_{j}\right)\right)+\left|S_{i}^{\prime} \backslash V\left(H_{j}\right)\right| \\
& =d_{G}\left(H_{j}\right)-\left((k-1)\left|S_{i}^{\prime} \cap X\right|-2\left|E\left(S_{i}^{\prime} \cap X\right)\right|-(k-1)\right)+\left|S_{i}^{\prime} \cap X\right|+1 \\
& =d_{G}\left(H_{j}\right)+2\left|E\left(S_{i}^{\prime} \cap X\right)\right|-(k-2)\left|S_{i}^{\prime} \cap X\right|+k \\
& \leqslant 3 k-(k-2)(k-1)+k=-k^{2}+7 k-2,
\end{aligned}
$$

which implies $k=5$. It is easy to verify that there is no triangle-free non-bipartite 4regular graph of order 9 , which implies $\left|S^{\prime}\right| \neq 9=2 k-1$, a contradiction. So Claim 12 holds.

Set $I_{i}^{\prime}=\left\{j \in\left[m_{3}\right]: S_{j}^{\prime} \cap V\left(H_{i}\right) \neq \emptyset\right\}$ for each $i \in[t]$ and $\mathscr{M}^{\prime}=\left\{\bigcup_{j \in I_{i}^{\prime}} S_{j}^{\prime}: i \in[t]\right\}$. Then any two sets in $\mathscr{M}^{\prime}$ are disjoint by Claim 12. By Lemma $20\left(\right.$ a),$d_{G}(U) \geqslant \lambda_{s}(G)$ for each $U \in \mathscr{M}^{\prime}$. Then, by Claim 11, we have

$$
\begin{aligned}
& 2\left(p+2+(k-1)\left(\left|\mathscr{M}^{\prime}\right|-p\right)\right) \\
= & 2|X| \geqslant 2\left|\bigcup_{U \in \mathscr{M}^{\prime}} \nabla(U)\right|=\sum_{U \in \mathscr{M}^{\prime}} d_{G}(U) \geqslant\left|\mathscr{M}^{\prime}\right| \lambda_{s}(G) \geqslant\left|\mathscr{M}^{\prime}\right|(2 k-2),
\end{aligned}
$$

which implies $p \leqslant \frac{2}{k-2}<1$, a contradiction. Hence Subcase 2.2.1 cannot occur.

Subcase 2.2.2 $\left|S^{\prime}\right| \geqslant 2 k$.

We have $\lambda_{s}(G)=\left|S^{\prime}\right| \geqslant 2 k$. If $s=4$, then $\lambda_{5}(G) \geqslant \lambda_{s}(G) \geqslant 2 k$. If $s \geqslant 5$, then $\lambda_{5}(G) \geqslant 2 k$ by the choice of $s$. Then $2 k p \leqslant p \lambda_{5}(G) \leqslant \sum_{i=1}^{p} d_{G}\left(H_{i}\right)+2|E(X)| \leqslant k(2+p)$, which implies $p \leqslant 2$.

Let

$$
\mathscr{N}=\left\{S_{i}^{\prime}: S_{i}^{\prime} \cap X \neq \emptyset \text { and } S_{i}^{\prime} \backslash(X \cup Y) \neq \emptyset, i \in\left[m_{3}\right]\right\} .
$$

By Claim 11, $\sum_{A \in \mathscr{N}}(|A \cap X|-|A \cap Y|)=\sum_{i=1}^{m_{3}}\left(\left|S_{i}^{\prime} \cap X\right|-\left|S_{i}^{\prime} \cap Y\right|\right)=|X|-|Y|=p+2$. Noting $|A \cap X|>|A \cap Y|$ for each $A \in \mathscr{N}$, we have $1 \leqslant|\mathscr{N}| \leqslant p+2$. Choose a set $S_{j_{1}}^{\prime} \in \mathscr{N}$. Without loss of generality, we assume $S_{j_{1}}^{\prime} \cap V\left(H_{1}\right) \neq \emptyset$. 
Suppose $p=2$. Then $E(X)=\emptyset$ and $2 k=\lambda_{5}(G)=d_{G}\left(H_{1}\right)=d_{G}\left(H_{2}\right)$. Hence $\lambda_{4}(G)=$ $\lambda_{5}(G)=2 k=\left|S^{\prime}\right|$. For each $u \in V(G)$ and each $i \in[p]$, we have $d_{G}\left(V\left(H_{i}\right) \cup\{u\}\right) \geqslant \lambda_{4}(G)$ and $d_{G}\left(V\left(H_{i}\right) \backslash\{u\}\right) \geqslant \lambda_{4}(G)$ by Lemma $20\left(\right.$ a), which implies $\left|\nabla(u) \cap \nabla\left(H_{i}\right)\right| \leqslant k-3$. Hence $\left|S_{j_{1}}^{\prime} \backslash V\left(H_{1}\right)\right| \geqslant 2$ and $\delta\left(G\left[S_{j_{1}}^{\prime} \cap V\left(H_{1}\right)\right]\right) \geqslant 2$, which implies $\left|S_{j_{1}}^{\prime} \cap V\left(H_{1}\right)\right| \geqslant 4$. Choose an edge $x_{2} y_{2} \in E\left(S_{j_{1}}^{\prime} \cap V\left(H_{1}\right)\right)$. Then $\left|S_{j_{1}}^{\prime} \backslash\left(V\left(H_{1}\right) \cup X\right)\right| \leqslant \mid S_{j_{1}}^{\prime} \backslash\left(N_{G\left[S_{j_{1}}^{\prime}\right]}\left(x_{2}\right) \cup\right.$ $\left.N_{G\left[S_{j_{1}}^{\prime}\right]}\left(y_{2}\right)\right) \mid=2$. It follows that $S_{j_{1}}^{\prime} \cap V\left(H_{2}\right)=\emptyset$. Noting that $d_{G\left[S_{j_{1}}^{\prime}\right]}\left(S_{j_{1}}^{\prime} \cap V\left(H_{1}\right)\right) \geqslant 2 k-4$ by Corollary 15, we have $\left|S_{j_{1}}^{\prime} \cap X\right| \geqslant\left|S_{j_{1}}^{\prime} \cap Y\right|+2$. Now

$$
\begin{aligned}
d_{G}\left(V\left(H_{1}\right) \cup S_{j_{1}}^{\prime}\right) & \leqslant d_{G}\left(H_{1}\right)-d_{G\left[S_{j_{1}}^{\prime}\right]}\left(V\left(H_{1}\right) \cap S_{j_{1}}^{\prime}\right)+\left|S_{j_{1}}^{\prime} \backslash V\left(H_{1}\right)\right| \\
& =2 k-(k-1)\left(\left|S_{j_{1}}^{\prime} \cap X\right|-\left|S_{j_{1}}^{\prime} \cap Y\right|\right)+\left|S_{j_{1}}^{\prime} \backslash V\left(H_{1}\right)\right| \\
& \leqslant 2 k-2(k-1)+2 k-4<2 k=\lambda_{4}(G),
\end{aligned}
$$

contradicting Lemma 20(a).

Thus $p=1$. Suppose $|\mathscr{N}|=1$. Then $\left|S_{j_{1}}^{\prime} \cap X\right|=\left|S_{j_{1}}^{\prime} \cap Y\right|+3$ and there is some $S_{j}^{\prime} \subseteq \overline{V\left(H_{1}\right) \cup S_{j_{1}}^{\prime}}$. We know by Claim 11 that $G\left[S^{\prime}\right]$ is bipartite. Hence there is some $S_{j^{\prime}}^{\prime} \subseteq V\left(H_{1}\right) \backslash S_{j_{1}}^{\prime}$. By Lemma 20(a), we have

$$
\begin{aligned}
\left|S^{\prime}\right|=\lambda_{s}(G) \leqslant d_{G}\left(V\left(H_{1}\right) \cup S_{j_{1}}^{\prime}\right) & \leqslant d_{G}\left(H_{1}\right)-d_{G\left[S_{j_{1}}^{\prime}\right]}\left(S_{j_{1}}^{\prime} \backslash V\left(H_{1}\right)\right)+\left|S_{j_{1}}^{\prime} \backslash V\left(H_{1}\right)\right| \\
& =d_{G}\left(H_{1}\right)+2\left|E\left(S_{j_{1}}^{\prime} \cap X\right)\right|-3(k-1)+\left|S_{j_{1}}^{\prime} \backslash V\left(H_{1}\right)\right| \\
& \leqslant 3 k-3(k-1)+\left|S_{j_{1}}^{\prime} \backslash V\left(H_{1}\right)\right| .
\end{aligned}
$$

Similarly, we can obtain $\left|S^{\prime}\right| \leqslant d_{G}\left(H_{1}-S_{j_{1}}^{\prime}\right) \leqslant 3+\left|S_{j_{1}}^{\prime} \cap V\left(H_{1}\right)\right|$. Then $2\left|S^{\prime}\right| \leqslant 6+\left|S_{j_{1}}^{\prime}\right|$, which implies $\left|S^{\prime}\right| \leqslant 6<2 k$, a contradiction.

Thus $|\mathscr{N}| \geqslant 2$. For each $S_{i}^{\prime} \in \mathscr{N}$, noting $\left|S_{i}^{\prime} \cap V\left(H_{1}\right)\right| \geqslant 2$, if $\left|S_{i}^{\prime} \backslash V\left(H_{1}\right)\right| \geqslant 2$, then, by Corollary 15, we have $d_{G\left[S_{i}^{\prime}\right]}\left(S_{i}^{\prime} \cap V\left(H_{1}\right)\right) \geqslant 2 k-4$, which implies that $\left|S_{i}^{\prime} \cap X\right|=1$ if $\left|S_{i}^{\prime} \cap X\right|=\left|S_{i}^{\prime} \cap Y\right|+1$. If $|\mathscr{N}|=3$, then $\left|S_{i}^{\prime} \cap X\right|=1$ for each $S_{i}^{\prime} \in \mathscr{N}$ and hence $d_{G}\left(V\left(H_{1}\right) \cup\left(\bigcup_{S_{i}^{\prime} \in \mathscr{N}} S_{i}^{\prime}\right)\right) \leqslant d_{G}\left(H_{1}\right)-3(k-2) \leqslant 6<\lambda_{s}(G)$, which contradicts Lemma 20(a). Thus $|\mathscr{N}|=2$. Assume $\mathscr{N}=\left\{S_{j_{1}}^{\prime}, S_{j_{2}}^{\prime}\right\}$ and $\left|S_{j_{1}}^{\prime} \cap X\right|=1$. We know that there is some $S_{j}^{\prime} \subseteq V(G) \backslash\left(V\left(H_{1}\right) \cup S_{j_{1}}^{\prime} \cup S_{j_{2}}^{\prime}\right)$. By Lemma $20(\mathrm{a})$,

$$
\begin{aligned}
|S|=\lambda_{s}(G) & \leqslant d_{G}\left(V\left(H_{1}\right) \cup S_{j_{1}}^{\prime} \cup S_{j_{2}}^{\prime}\right) \\
& \leqslant d_{G}\left(H_{1}\right)-d_{G\left[S_{j_{2}}^{\prime}\right]}\left(S_{j_{2}}^{\prime} \cap V\left(H_{1}\right)\right)+\left|S_{j_{2}}^{\prime} \backslash V\left(H_{1}\right)\right|-(k-2) \\
& =d_{G}\left(H_{1}\right)+2\left|E\left(S_{j_{2}}^{\prime} \cap X\right)\right|-2(k-1)+\left|S_{j_{2}}^{\prime} \backslash V\left(H_{1}\right)\right|-(k-2) \\
& \leqslant 3 k-3 k+4+\left|S_{j_{2}}^{\prime} \backslash V\left(H_{1}\right)\right| .
\end{aligned}
$$

Similarly, we can obtain $\left|S^{\prime}\right| \leqslant d_{G}\left(\left(V\left(H_{1}\right) \cup S_{j_{1}}^{\prime}\right) \backslash S_{j_{2}}^{\prime}\right) \leqslant 4+\left|S_{j_{2}}^{\prime} \cap V\left(H_{1}\right)\right|$. Then $2\left|S^{\prime}\right| \leqslant$ $8+\left|S_{j_{2}}^{\prime}\right|$, which implies $\left|S^{\prime}\right| \leqslant 8<2 k$, a contradiction.

\section{References}

[1] B. Andrásfai, P. Erdős, and V. T. Sós, On the connection between chromatic number, maximal clique and minimal degree of a graph, Discrete Math., 8:205-218, 1974. 
[2] N. Biggs, Algebraic Graph Theory, pages 36-38. Cambridge University Press, 1993.

[3] O. Chan, C.C. Chen, and Q. Yu, On 2-extendable obelian Cayley graphs, Discrete Math., 146:19-32, 1995.

[4] C.C. Chen, J. Liu, and Q. Yu, On the classification of 2-extendable Cayley graphs on dihedral groups, Australas. J. Combin., 6:209-219, 1992.

[5] R. Diestel, Graph Theory, page 41. Springer, 2006.

[6] J. Fàbrega, and M.A. Fiol, Extraconnectivity of graphs with large girth, Discrete Math., 127:163-170, 1994.

[7] O. Favaron, On k-factor-critical graphs, Discuss. Math. Graph Theory, 16:41-51, 1996.

[8] O. Favaron, Extendability and factor-criticality, Discrete Math., 213:115-122, 2000.

[9] T. Gallai, Neuer Beweis eines Tutte-schen Satzes, Magyar Tud. Akad. Matem. Kut. Int. Közl., 8:135-139, 1963.

[10] J. Heuvel, and B. Jacskon, On the edge connectivity, hamiltonicity, and toughness of vertex transitive graphs, J. Combin. Theory Ser. B, 77:138-149, 1999.

[11] A. Holtkamp, D. Meierling, and L.P. Montejano, $k$-restricted edge-connectivity in triangle-free graphs, Discrete Appl. Math., 160:1345-1355, 2012.

[12] L. Lovász, On the structure of factorizable graphs, Acta Math. Acad. Sci. Hungar., 23:179-195 1972.

[13] L. Lovász, and M.D. Plummer, Matching Theory, page 207. North-Holland, 1986.

[14] W. Mader, Minimale n-fach kantenzusammenhängenden Graphen, Math. Ann., 191:21-28, 1971.

[15] W. Mantel, Problem 28, Wiskundige Opgaven, 10:60-61, 1907.

[16] Š. Miklavič, and P. Šparl, On extendability of Cayley graphs, Filomat, 23:93-101, 2009.

[17] M.D. Plummer, On n-extendable graphs, Discrete Math., 31:201-210, 1980.

[18] R. Tindell, Connectivity of Cayley graphs, In D.Z. Du, D.F. Hsu (Eds.). Combinatorial Network Theory, pages 41-64. Kluwer, 1996.

[19] M.E. Watkins, Connectivity of transitive graphs, J. Combin. Theory, 8:23-29, 1970.

[20] M. Yang, Z. Zhang, C. Qin, and X. Guo, On super 2-restricted and 3-restricted edge-connected vertex transitive graphs, Discrete Math., 311:2683-2689, 2011.

[21] Q. Yu, Characterizations of various matching extensions in graphs, Australas. J. Combin., 7:55-64, 1993.

[22] H. Zhang, and W. Sun, 3-Factor-criticality of vertex-transitive graphs, J. Graph Theory, 81:262-271, 2016. 